\title{
Morrey Meets Herz with Variable Exponent and Applications to Commutators of Homogeneous Fractional Integrals with Rough Kernels
}

\author{
Hongbin Wang, ${ }^{1,2}$ Jiajia Wang, ${ }^{3}$ and Zunwei Fu ${ }^{4}$ \\ ${ }^{1}$ School of Mathematical Sciences, University of Chinese Academy of Sciences, Beijing 100049, China \\ ${ }^{2}$ School of Mathematics and Statistics, Shandong University of Technology, Zibo 255049, China \\ ${ }^{3}$ Department of Mathematics, Linyi University, Linyi 276005, China \\ ${ }^{4}$ Department of Mathematics, The University of Suwon, Wau-ri, Bongdam-eup, Hwaseong-si, \\ Gyeonggi-do 445-743, Republic of Korea
}

Correspondence should be addressed to Zunwei Fu; zwfu@mail.bnu.edu.cn

Received 25 April 2017; Accepted 11 July 2017; Published 25 September 2017

Academic Editor: Alberto Fiorenza

Copyright (c) 2017 Hongbin Wang et al. This is an open access article distributed under the Creative Commons Attribution License, which permits unrestricted use, distribution, and reproduction in any medium, provided the original work is properly cited.

We define the new central Morrey space with variable exponent and investigate its relation to the Morrey-Herz spaces with variable exponent. As applications, we obtain the boundedness of the homogeneous fractional integral operator $T_{\Omega, \sigma}$ and its commutator $\left[b, T_{\Omega, \sigma}\right]$ on Morrey-Herz space with variable exponent, where $\Omega \in L^{s}\left(S^{n-1}\right)$ for $s \geq 1$ is a homogeneous function of degree zero, $0<\sigma<n$, and $b$ is a BMO function.

\section{Introduction}

The Morrey spaces first appeared in 1938 in the work of Morrey [1] in relation to some problems in partial differential equations. In [2] the authors introduced central Morrey spaces. The Herz spaces are a class of function spaces introduced by Herz in the study of absolutely convergent Fourier transforms in 1968; see [3]. The complete theory of Herz spaces for the case of general indexes was established by Lu et al. in 2008; see [4]. Recently, Lu and Xu defined the homogeneous Morrey-Herz spaces in [5].

The theory of function spaces with variable exponent was extensively studied by researchers since the work of Kováčik and Rákosník [6] appeared in 1991; see [7, 8] and the references therein. Many applications of these spaces were given, for example, in the modeling of electrorheological fluids [9], in the study of image processing [10], and in differential equations with nonstandard growth [11]. In 2009, Izuki established the Herz spaces with variable exponent and Morrey-Herz spaces with variable exponent; see [12, 13]. In [14], the authors introduced the nonhomogeneous central Morrey spaces of variable exponent.
Suppose that $S^{n-1}$ denotes the unit sphere in $\mathbb{R}^{n}(n \geq$ 2 ) equipped with normalized Lebesgue measure. Let $\Omega \in$ $L^{s}\left(S^{n-1}\right)$ for $s \geq 1$ be a homogeneous function of degree zero. For $0<\sigma<n$, the homogeneous fractional integral operator $T_{\Omega, \sigma}$ is defined by

$$
T_{\Omega, \sigma} f(x)=\int_{\mathbb{R}^{n}} \frac{\Omega(x-y)}{|x-y|^{n-\sigma}} f(y) d y .
$$

Denote $T_{\sigma}=T_{\Omega, \sigma}$ when $\Omega \equiv 1$. In 1971, Muckenhoupt and Wheeden [15] gave the weighted $\left(L^{p}, L^{q}\right)$ boundedness of $T_{\Omega, \sigma}$ with power weights. Recently, Tan and Liu [16] gave some boundedness of $T_{\Omega, \sigma}$ on function spaces with variable exponent. For the applications of fractional integral operator in fractional order system, we choose to refer to [17].

Let $b$ be a locally integrable function; the commutator of homogeneous fractional integral operator $\left[b, T_{\Omega, \sigma}\right]$ is defined by

$$
\begin{aligned}
& {\left[b, T_{\Omega, \sigma}\right] f(x)} \\
& \quad=\int_{\mathbb{R}^{n}} \frac{\Omega(x-y)}{|x-y|^{n-\sigma}}(b(x)-b(y)) f(y) d y .
\end{aligned}
$$


Motivated by the above references, in the present paper we will study the boundedness for the homogeneous fractional integral operator $T_{\Omega, \sigma}$ and its commutator $\left[b, T_{\Omega, \sigma}\right]$ on the Morrey-Herz space with variable exponent.

Let us explain the outline of this article. In Section 2, we first briefly recall some standard notations and lemmas in variable Lebesgue spaces. In Section 3, we will give the relation between central Morrey spaces, Herz spaces, and Morrey-Herz spaces with variable exponent. We will establish the boundedness for the homogeneous fractional integral operator $T_{\Omega, \sigma}$ and its commutator $\left[b, T_{\Omega, \sigma}\right]$ on the MorreyHerz space with variable exponent in Sections 4 and 5.

\section{Variable Lebesgue Spaces}

Given an open set $E \subset \mathbb{R}^{n}$ and a measurable function $p(\cdot)$ : $E \rightarrow[1, \infty), L^{p(\cdot)}(E)$ denotes the set of measurable functions $f$ on $E$ such that for some $\lambda>0$

$$
\int_{E}\left(\frac{|f(x)|}{\lambda}\right)^{p(x)} d x<\infty
$$

This set becomes a Banach function space when equipped with the Luxemburg-Nakano norm

$$
\|f\|_{L^{p(\cdot)}(E)}=\inf \left\{\lambda>0: \int_{E}\left(\frac{|f(x)|}{\lambda}\right)^{p(x)} d x \leq 1\right\} .
$$

These spaces are referred to as variable $L^{p}$ spaces, since they generalized the standard $L^{p}$ spaces: if $p(x)=p$ is constant, then $L^{p(\cdot)}(E)$ is isometrically isomorphic to $L^{p}(E)$.

The space $L_{\text {loc }}^{p(\cdot)}(E)$ is defined by

$$
\begin{aligned}
& L_{\text {loc }}^{p^{(\cdot)}}(E) \\
& \quad:=\left\{f: f \in L^{p(\cdot)}(F) \forall \text { compact subsets } F \subset E\right\} .
\end{aligned}
$$

Define $\mathscr{P}^{0}(E)$ to be set of $p(\cdot): E \rightarrow(0, \infty)$ such that

$$
\begin{aligned}
& p^{-}=\operatorname{ess} \inf \{p(x): x \in E\}>0, \\
& p^{+}=\operatorname{ess} \sup \{p(x): x \in E\}<\infty .
\end{aligned}
$$

Define $\mathscr{P}(E)$ to be set of $p(\cdot): E \rightarrow[1, \infty)$ such that

$$
\begin{aligned}
& p^{-}=\operatorname{essinf}\{p(x): x \in E\}>1, \\
& p^{+}=\operatorname{ess} \sup \{p(x): x \in E\}<\infty .
\end{aligned}
$$

Denote $p^{\prime}(x)=p(x) /(p(x)-1)$. In addition, we denote the Lebesgue measure and the characteristic function of a measurable set $A \subset \mathbb{R}^{n}$ by $|A|$ and $\chi_{A}$, respectively. The notation $f \approx g$ means that there exist constants $C_{1}, C_{2}>0$ such that $C_{1} g \leq f \leq C_{2} g$.

In variable $L^{p}$ spaces there are some important lemmas as follows.
Lemma 1 (see $[18])$. If $p(\cdot) \in \mathscr{P}\left(\mathbb{R}^{n}\right)$ and satisfies

$$
\begin{aligned}
& |p(x)-p(y)| \leq \frac{C}{-\log (|x-y|)}, \quad|x-y| \leq \frac{1}{2}, \\
& |p(x)-p(y)| \leq \frac{C}{\log (|x|+e)}, \quad|y| \geq|x|,
\end{aligned}
$$

then $p(\cdot) \in \mathscr{B}\left(\mathbb{R}^{n}\right)$; that is, the Hardy-Littlewood maximal operator $M$ is bounded on $L^{p(\cdot)}\left(\mathbb{R}^{n}\right)$.

Lemma 2 (see [6]). Let $p(\cdot) \in \mathscr{P}\left(\mathbb{R}^{n}\right)$. If $f \in L^{p(\cdot)}\left(\mathbb{R}^{n}\right)$ and $g \in L^{p^{\prime}(\cdot)}\left(\mathbb{R}^{n}\right)$, then $f g$ is integrable on $\mathbb{R}^{n}$ and

$$
\int_{\mathbb{R}^{n}}|f(x) g(x)| d x \leq r_{p}\|f\|_{L^{p(\cdot)}\left(\mathbb{R}^{n}\right)}\|g\|_{L^{p^{\prime}(\cdot)}\left(\mathbb{R}^{n}\right)},
$$

where

$$
r_{p}=1+\frac{1}{p^{-}}-\frac{1}{p^{+}} .
$$

This inequality is named the generalized Hölder inequality with respect to the variable $L^{p}$ spaces.

Lemma 3 (see [19]). Let $p(\cdot) \in \mathscr{B}\left(\mathbb{R}^{n}\right)$. Then there exists a positive constant $C$ such that for all balls $B$ in $\mathbb{R}^{n}$ and all measurable subsets $S \subset B$

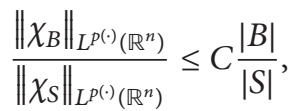

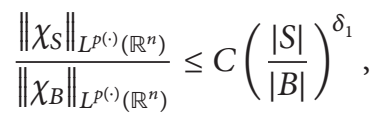

$$
\begin{aligned}
& \frac{\left\|\chi_{S}\right\|_{L^{p^{\prime}(\cdot)}\left(\mathbb{R}^{n}\right)}}{\left\|\chi_{B}\right\|_{L^{p^{\prime}(\cdot)}\left(\mathbb{R}^{n}\right)}} \leq C\left(\frac{|S|}{|B|}\right)^{\delta_{2}},
\end{aligned}
$$

where $0<\delta_{1}, \delta_{2}<1$ depend on $p(\cdot)$.

Throughout this paper $\delta_{2}$ will change with $p(\cdot)$.

Lemma 4 (see [19]). Suppose $p(\cdot) \in \mathscr{B}\left(\mathbb{R}^{n}\right)$. Then there exists a constant $C>0$ such that for all balls $B$ in $\mathbb{R}^{n}$

$$
\frac{1}{|B|}\left\|\chi_{B}\right\|_{L^{p(\cdot)}\left(\mathbb{R}^{n}\right)}\left\|\chi_{B}\right\|_{L^{p^{(\cdot)}\left(\mathbb{R}^{n}\right)}} \leq C .
$$

\section{The Relation between Central Morrey Spaces, Herz Spaces, and Morrey-Herz Spaces with Variable Exponent}

Let $B_{k}=\left\{x \in \mathbb{R}^{n}:|x| \leq 2^{k}\right\}$ and $A_{k}=B_{k} \backslash B_{k-1}$ for $k \in \mathbb{Z}$. Denote $\mathbb{Z}_{+}$and $\mathbb{N}$ as the sets of all positive and nonnegative integers, $\chi_{k}=\chi_{A_{k}}$ for $k \in \mathbb{Z}, \tilde{\chi}_{k}=\chi_{k}$ if $k \in \mathbb{Z}_{+}$and $\tilde{\chi}_{0}=\chi_{B_{0}}$.

We can give the following definitions of function spaces with variable exponent.

Definition 5. Let $q(\cdot) \in \mathscr{P}\left(\mathbb{R}^{n}\right)$ and $\lambda \in \mathbb{R}$. The homogeneous central Morrey space with variable exponent $\dot{\mathscr{B}}^{q(\cdot), \lambda}\left(\mathbb{R}^{n}\right)$ is defined by

$$
\dot{\mathscr{B}}^{q(\cdot), \lambda}\left(\mathbb{R}^{n}\right)=\left\{f \in L_{\text {loc }}^{q(\cdot)}\left(\mathbb{R}^{n}\right):\|f\|_{\dot{\mathscr{B}}^{q(\cdot), \lambda}\left(\mathbb{R}^{n}\right)}<\infty\right\},
$$


where

$$
\|f\|_{\dot{\dot{B}^{q}}(\cdot), \lambda\left(\mathbb{R}^{n}\right)}=\sup _{L \in \mathbb{Z}} 2^{-L \lambda} \sum_{k=-\infty}^{L}\left\|f \chi_{k}\right\|_{L^{q(\cdot)}\left(\mathbb{R}^{n}\right)} .
$$

The nonhomogeneous central Morrey space with variable exponent $\mathscr{B}^{q(\cdot), \lambda}\left(\mathbb{R}^{n}\right)$ is defined by

$$
\mathscr{B}^{q(\cdot), \lambda}\left(\mathbb{R}^{n}\right)=\left\{f \in L_{\text {loc }}^{q(\cdot)}\left(\mathbb{R}^{n}\right):\|f\|_{\mathscr{B}^{q(\cdot), \lambda}\left(\mathbb{R}^{n}\right)}<\infty\right\},
$$

where

$$
\|f\|_{\mathscr{B}^{q(\cdot), \lambda\left(\mathbb{R}^{n}\right)}}=\sup _{L \in \mathbb{Z}} 2^{-L \lambda} \sum_{k=0}^{L}\left\|f \widetilde{\chi}_{k}\right\|_{L^{q(\cdot)}\left(\mathbb{R}^{n}\right)} .
$$

Definition 6 (see [19]). Let $\alpha \in \mathbb{R}, 0<p \leq \infty$, and $q(\cdot) \in$ $\mathscr{P}\left(\mathbb{R}^{n}\right)$. The homogeneous Herz space with variable exponent $\dot{K}_{q(\cdot)}^{\alpha, p}\left(\mathbb{R}^{n}\right)$ is defined by

$$
\begin{aligned}
\dot{K}_{q(\cdot)}^{\alpha, p}\left(\mathbb{R}^{n}\right) & \\
\quad= & \left\{f \in L_{\text {loc }}^{q(\cdot)}\left(\mathbb{R}^{n} \backslash\{0\}\right):\|f\|_{\dot{K}_{q(\cdot)}^{\alpha, p}\left(\mathbb{R}^{n}\right)}<\infty\right\},
\end{aligned}
$$

where

$$
\|f\|_{\dot{K}_{q(\cdot)}^{\alpha, p}\left(\mathbb{R}^{n}\right)}=\left\{\sum_{k=-\infty}^{\infty} 2^{k \alpha p}\left\|f \chi_{k}\right\|_{L^{q(\cdot)}\left(\mathbb{R}^{n}\right)}^{p}\right\}^{1 / p} .
$$

The nonhomogeneous Herz space with variable exponent $K_{q(\cdot)}^{\alpha, p}\left(\mathbb{R}^{n}\right)$ is defined by

$$
K_{q(\cdot)}^{\alpha, p}\left(\mathbb{R}^{n}\right)=\left\{f \in L_{\text {loc }}^{q(\cdot)}\left(\mathbb{R}^{n}\right):\|f\|_{K_{q(\cdot)}^{\alpha, p}\left(\mathbb{R}^{n}\right)}<\infty\right\},
$$

where

$$
\|f\|_{K_{q(\cdot)}^{\alpha, p}\left(\mathbb{R}^{n}\right)}=\left\{\sum_{k=0}^{\infty} 2^{k \alpha p}\left\|f \tilde{\chi}_{k}\right\|_{L^{q(\cdot)}\left(\mathbb{R}^{n}\right)}^{p}\right\}^{1 / p} .
$$

Definition 7. Let $\alpha \in \mathbb{R}, 0<p \leq \infty, q(\cdot) \in \mathscr{P}\left(\mathbb{R}^{n}\right)$, and $0 \leq$ $\lambda<\infty$. The homogeneous Morrey-Herz space with variable exponent $M \dot{K}_{p, q(\cdot)}^{\alpha, \lambda}\left(\mathbb{R}^{n}\right)$ (see [13]) is defined by

$$
\begin{aligned}
M & \dot{K}_{p, q(\cdot)}^{\alpha, \lambda}\left(\mathbb{R}^{n}\right) \\
& =\left\{f \in L_{\text {loc }}^{q(\cdot)}\left(\mathbb{R}^{n} \backslash\{0\}\right):\|f\|_{M \dot{K}_{p, q(\cdot)}^{\alpha, \lambda}\left(\mathbb{R}^{n}\right)}<\infty\right\},
\end{aligned}
$$

where

$$
\begin{aligned}
&\|f\|_{M \dot{K}_{p, q(\cdot)}^{\alpha, \lambda}\left(\mathbb{R}^{n}\right)} \\
&=\sup _{L \in \mathbb{Z}} 2^{-L \lambda}\left\{\sum_{k=-\infty}^{L} 2^{k \alpha p}\left\|f \chi_{k}\right\|_{L^{q \cdot(\cdot)}\left(\mathbb{R}^{n}\right)}^{p}\right\}^{1 / p} .
\end{aligned}
$$

The nonhomogeneous Morrey-Herz space with variable exponent $M K_{p, q(\cdot)}^{\alpha, \lambda}\left(\mathbb{R}^{n}\right)$ is defined by

$$
\begin{aligned}
& M K_{p, q(\cdot)}^{\alpha, \lambda}\left(\mathbb{R}^{n}\right) \\
& \quad=\left\{f \in L_{\text {loc }}^{q(\cdot)}\left(\mathbb{R}^{n}\right):\|f\|_{M K_{p, q(\cdot)}^{\alpha, \lambda}\left(\mathbb{R}^{n}\right)}<\infty\right\},
\end{aligned}
$$

where

$$
\|f\|_{M K_{p, q(\cdot)}^{\alpha, \lambda}\left(\mathbb{R}^{n}\right)}=\sup _{L \in \mathbb{Z}} 2^{-L \lambda}\left\{\sum_{k=0}^{L} 2^{k \alpha p}\left\|f \tilde{\chi}_{k}\right\|_{L^{q(\cdot)}\left(\mathbb{R}^{n}\right)}^{p}\right\}^{1 / p} .
$$

Remark 8. If $\alpha=0, p=1$, then $M \dot{K}_{1, q(\cdot)}^{0, \lambda}\left(\mathbb{R}^{n}\right)=\dot{\mathscr{B}}^{q(\cdot), \lambda}\left(\mathbb{R}^{n}\right)$ and $M K_{1, q(\cdot)}^{0, \lambda}\left(\mathbb{R}^{n}\right)=\mathscr{B}^{q(\cdot), \lambda}\left(\mathbb{R}^{n}\right)$.

Remark 9. If $\lambda=0$, then $M \dot{K}_{p, q(\cdot)}^{\alpha, 0}\left(\mathbb{R}^{n}\right)=\dot{K}_{q(\cdot)}^{\alpha, p}\left(\mathbb{R}^{n}\right)$ and $M K_{p, q(\cdot)}^{\alpha, 0}\left(\mathbb{R}^{n}\right)=K_{q(\cdot)}^{\alpha, p}\left(\mathbb{R}^{n}\right)$.

Remark 10. If $q(\cdot)=q$ is a constant, then $M \dot{K}_{p, q(\cdot)}^{\alpha, \lambda}\left(\mathbb{R}^{n}\right)=$ $M \dot{K}_{p, q}^{\alpha, \lambda}\left(\mathbb{R}^{n}\right)$ and $M K_{p, q(\cdot)}^{\alpha, \lambda}\left(\mathbb{R}^{n}\right)=M K_{p, q}^{\alpha, \lambda}\left(\mathbb{R}^{n}\right)$.

\section{Estimate for the Homogeneous Fractional Integral Operator}

In this section we will prove the boundedness of the homogeneous fractional integral operators $T_{\Omega, \sigma}$ on Morrey-Herz spaces with variable exponent.

Theorem 11. Suppose that $0<v \leq 1,0<\sigma<n-v$, $q_{1}(\cdot) \in \mathscr{P}\left(\mathbb{R}^{n}\right)$ satisfies conditions (8) in Lemma 1 with $q_{1}^{+}<$ $n / \sigma, 1 / q_{1}(x)-1 / q_{2}(x)=\sigma / n, \Omega \in L^{s}\left(S^{n-1}\right)\left(s>q_{1}^{\prime-}\right)$. Let $0<p_{1} \leq p_{2}<\infty$ and $0<\lambda<\alpha<n \delta_{2}-v-n / s$ (or $\left.0<\lambda<\alpha_{2} \leq \alpha_{1}<n \delta_{2}-v-n / s\right)$. Then $T_{\Omega, \sigma}$ is bounded from $M \dot{K}_{q_{1}(\cdot)}^{\alpha, p_{1}}\left(\mathbb{R}^{n}\right)\left(\right.$ or $\left.M K_{q_{1}(\cdot)}^{\alpha_{1}, p_{1}}\left(\mathbb{R}^{n}\right)\right)$ to $M \dot{K}_{q_{2}(\cdot)}^{\alpha, p_{2}}\left(\mathbb{R}^{n}\right)$ (or $\left.M K_{q_{2}(\cdot)}^{\alpha_{2}, p_{2}}\left(\mathbb{R}^{n}\right)\right)$.

To prove Theorem 11, we need the following lemmas.

Lemma 12 (see [15]). If $a>0,1 \leq s \leq \infty, 0<d \leq s$, and $-n+(n-1) d / s<v<\infty$, then

$$
\begin{aligned}
& \left(\int_{|y| \leq a|x|}|y|^{\nu}|\Omega(x-y)|^{d} d y\right)^{1 / d} \\
& \quad \leq C|x|^{(\gamma+n) / d}\|\Omega\|_{L^{s}\left(S^{n-1}\right)} .
\end{aligned}
$$

Lemma 13 (see [20]). Define a variable exponent $\widetilde{q}(\cdot)$ by $1 / p(x)=1 / \widetilde{q}(x)+1 / q\left(x \in \mathbb{R}^{n}\right)$. Then we have

$$
\|f g\|_{L^{p \cdot(\cdot)}\left(\mathbb{R}^{n}\right)} \leq C\|f\|_{L^{\tilde{q} \cdot(\cdot)}\left(\mathbb{R}^{n}\right)}\|g\|_{L^{q}\left(\mathbb{R}^{n}\right)},
$$

for all measurable functions $f$ and $g$.

Lemma 14 (see $[8])$. Let $p(\cdot) \in \mathscr{P}\left(\mathbb{R}^{n}\right)$ satisfy conditions (8) in Lemma 1. Then

$$
\left\|\chi_{Q}\right\|_{L^{p(\cdot)}\left(\mathbb{R}^{n}\right)} \approx \begin{cases}|Q|^{1 / p(x)} & \text { if }|Q| \leq 2^{n}, x \in Q, \\ |Q|^{1 / p(\infty)} & \text { if }|Q| \geq 1,\end{cases}
$$

for every cube (or ball) $Q \subset \mathbb{R}^{n}$, where $p(\infty)=\lim _{x \rightarrow \infty} p(x)$.

Proof of Theorem 11. We only prove the homogeneous case. Similar to the method of [21], it is easy to prove that 
4

Journal of Function Spaces

$M K_{q_{2}(\cdot)}^{\alpha_{1}, p_{2}}\left(\mathbb{R}^{n}\right) \subset M K_{q_{2}(\cdot)}^{\alpha_{2}, p_{2}}\left(\mathbb{R}^{n}\right)$ for $0<\alpha_{2} \leq \alpha_{1}$. So the nonhomogeneous case can be proved in the same way. Let $f \in M \dot{K}_{q_{1}(\cdot)}^{\alpha, p_{1}}\left(\mathbb{R}^{n}\right)$. Denote $f_{j}=f \chi_{j}$ for each $j \in \mathbb{Z}$; then we have $f(x)=\sum_{j=-\infty}^{\infty} f_{j}(x)$. Note that $p_{1} \leq p_{2}$; we have

$$
\begin{aligned}
& \left\|T_{\Omega, \sigma}(f)\right\|_{M \dot{K}_{q_{2}(\cdot)}^{\alpha, p_{2}\left(\mathbb{R}^{n}\right)}}^{p_{1}}=\sup _{L \in \mathbb{Z}} 2^{-L \lambda p_{1}} \\
& \cdot\left\{\sum_{k=-\infty}^{L} 2^{k \alpha p_{2}}\left\|T_{\Omega, \sigma}(f) \chi_{k}\right\|_{L^{q_{2}(\cdot)}\left(\mathbb{R}^{n}\right)}^{p_{2}}\right\}^{p_{1} / p_{2}} \\
& \leq \sup _{L \in \mathbb{Z}} 2^{-L \lambda p_{1}} \sum_{k=-\infty}^{L} 2^{k \alpha p_{1}}\left\|T_{\Omega, \sigma}(f) \chi_{k}\right\|_{L^{q_{2}(\cdot)}\left(\mathbb{R}^{n}\right)}^{p_{1}} \leq C \\
& \left.\cdot \sup _{L \in \mathbb{Z}} 2^{-L \lambda p_{1}}\right)^{p_{1}}+C \\
& \cdot \sum_{k=-\infty}^{L} 2^{k \alpha p_{1}}\left(\sum_{j=-\infty}^{k-2}\left\|T_{\Omega, \sigma}\left(f_{j}\right) \chi_{k}\right\|_{L^{q_{2}(\cdot)}\left(\mathbb{R}^{n}\right)}\right)^{p^{2}}+C \\
& \cdot \sup _{L \in \mathbb{Z}} 2^{-L \lambda p_{1}} \\
& \quad \sum_{k=-\infty}^{L} 2^{k \alpha p_{1}}\left(\sum_{j=k-1}^{\infty}\left\|T_{\Omega, \sigma}\left(f_{j}\right) \chi_{k}\right\|_{L^{q_{2}(\cdot)}\left(\mathbb{R}^{n}\right)}\right)^{p_{1}}=: I_{1} \\
& +I_{2} \cdot
\end{aligned}
$$

We first estimate $I_{1}$. For each $k \in \mathbb{Z}, j \leq k-2$, and a.e. $x \in A_{k}$, using the generalized Hölder inequality, we have

$$
\begin{aligned}
& \left|T_{\Omega, \sigma}\left(f_{j}\right)(x)\right| \leq C \int_{B_{j}} \frac{|\Omega(x-y)|}{|x-y|^{n-\sigma}}\left|f_{j}(y)\right| d y \\
& \quad \leq C 2^{k(\sigma-n)} \int_{B_{j}}|\Omega(x-y)|\left|f_{j}(y)\right| d y \\
& \quad \leq C 2^{k(\sigma-n)}\left\|\Omega(x-\cdot) \chi_{j}(\cdot)\right\|_{L^{q_{1}^{\prime}(\cdot)}\left(\mathbb{R}^{n}\right)}\left\|f_{j}\right\|_{L^{q_{1}(\cdot)}\left(\mathbb{R}^{n}\right)} .
\end{aligned}
$$

Noting $s>q_{1}^{\prime-}$, we denote $\widetilde{q_{1}^{\prime}}(\cdot)>1$ and $1 / q_{1}^{\prime}(x)=$ $1 / \widetilde{q_{1}^{\prime}}(x)+1 / s$. By Lemmas 12 and 13 we have

$$
\begin{aligned}
& \left\|\Omega(x-\cdot) \chi_{j}(\cdot)\right\|_{L^{q_{1}^{\prime}(\cdot)}\left(\mathbb{R}^{n}\right)} \\
& \leq\left\|\Omega(x-\cdot) \chi_{j}(\cdot)\right\|_{L^{s}\left(\mathbb{R}^{n}\right)}\left\|\chi_{j}(\cdot)\right\|_{L^{\widetilde{q_{1}^{T}}(\cdot)}\left(\mathbb{R}^{n}\right)} \\
& \leq\left\|\Omega(x-\cdot) \chi_{j}(\cdot)\right\|_{L^{s}\left(\mathbb{R}^{n}\right)}\left\|\chi_{B_{j}}\right\|_{L^{q_{1}^{\top} \cdot(\cdot)}\left(\mathbb{R}^{n}\right)} \\
& \leq C 2^{-j v}\left(\int_{A_{j}}|\Omega(x-y)|^{s}|y|^{s v} d y\right)^{1 / s}\left\|\chi_{B_{j}}\right\|_{L^{\tau_{1}^{\prime}(\cdot)}\left(\mathbb{R}^{n}\right)} \\
& \leq C 2^{-j v} 2^{k(v+n / s)}\|\Omega\|_{L^{s}\left(S^{n-1}\right)}\left\|\chi_{B_{j}}\right\|_{L^{q_{1}^{\top} \cdot(\cdot)}\left(\mathbb{R}^{n}\right)} .
\end{aligned}
$$

When $\left|B_{j}\right| \leq 2^{n}$ and $x_{j} \in B_{j}$, by Lemma 14 , we have

$$
\left\|\chi_{B_{j}}\right\|_{L^{q_{1}^{\prime} \cdot(\cdot)}\left(\mathbb{R}^{n}\right)} \approx\left|B_{j}\right|^{1 / \widetilde{q_{1}^{\prime}}\left(x_{j}\right)} \approx\left\|\chi_{B_{j}}\right\|_{L^{q_{1}^{\prime} \cdot(\cdot)}\left(\mathbb{R}^{n}\right)}\left|B_{j}\right|^{-1 / s} .
$$

When $\left|B_{j}\right| \geq 1$ we have

$$
\left\|\chi_{B_{j}}\right\|_{L^{\tau_{1}^{\prime} \cdot(\cdot)}\left(\mathbb{R}^{n}\right)} \approx\left|B_{j}\right|^{1 / \widetilde{q_{1}^{\prime}}(\infty)} \approx\left\|\chi_{B_{j}}\right\|_{L^{q_{1}^{\prime} \cdot(\cdot)\left(\mathbb{R}^{n}\right)}}\left|B_{j}\right|^{-1 / s} .
$$

Since

$$
T_{\sigma}\left(\chi_{B_{k}}\right)(x) \geq \int_{B_{k}} \frac{d y}{|x-y|^{n-\sigma}} \chi_{B_{k}}(x) \geq C 2^{k \sigma} \chi_{B_{k}}(x),
$$

by Lemmas 3 and 4, we have

$$
\begin{aligned}
& \left\|T_{\Omega, \sigma}\left(f_{j}\right) \chi_{k}\right\|_{L^{q_{2}(\cdot)}\left(\mathbb{R}^{n}\right)} \\
& \leq C 2^{k(\sigma-n)} 2^{-j v} 2^{k(v+n / s)}\|\Omega\|_{L^{s}\left(S^{n-1}\right)}\left\|f_{j}\right\|_{L^{q_{1}(\cdot)}\left(\mathbb{R}^{n}\right)} \\
& \cdot\left\|\chi_{B_{j}}\right\|_{L^{\widetilde{q_{1}^{\prime}} \cdot(\cdot)}\left(\mathbb{R}^{n}\right)}\left\|\chi_{B_{k}}\right\|_{L^{q_{2}(\cdot)}\left(\mathbb{R}^{n}\right)} \\
& \leq C 2^{k(\sigma-n)} 2^{-j v} 2^{k(v+n / s)}\|\Omega\|_{L^{s}\left(S^{n-1}\right)}\left\|f_{j}\right\|_{L^{q_{1}(\cdot)}\left(\mathbb{R}^{n}\right)} \\
& \cdot\left\|\chi_{B_{j}}\right\|_{L^{q_{1}^{\prime}(\cdot)}\left(\mathbb{R}^{n}\right)}\left|B_{j}\right|^{-1 / s}\left\|\chi_{B_{k}}\right\|_{L^{q_{2}(\cdot)}\left(\mathbb{R}^{n}\right)} \\
& \leq C 2^{-k n} 2^{-j v} 2^{k(v+n / s)}\|\Omega\|_{L^{s}\left(S^{n-1}\right)}\left\|f_{j}\right\|_{L^{q_{1}(\cdot)}\left(\mathbb{R}^{n}\right)} \\
& \cdot\left\|\chi_{B_{j}}\right\|_{L^{q_{1}^{\prime}(\cdot)}\left(\mathbb{R}^{n}\right)}\left|B_{j}\right|^{-1 / s}\left\|T_{\sigma}\left(\chi_{B_{k}}\right)\right\|_{L^{q_{2}(\cdot)}\left(\mathbb{R}^{n}\right)} \\
& \leq C 2^{-k n+(k-j)(\nu+n / s)}\|\Omega\|_{L^{s}\left(S^{n-1}\right)}\left\|f_{j}\right\|_{L^{q_{1}(\cdot)}\left(\mathbb{R}^{n}\right)} \\
& \cdot\left\|\chi_{B_{j}}\right\|_{L^{q_{1}^{\prime}(\cdot)}\left(\mathbb{R}^{n}\right)}\left\|\chi_{B_{k}}\right\|_{L^{q_{1}(\cdot)}\left(\mathbb{R}^{n}\right)} \\
& \leq C 2^{(k-j)(\nu+n / s)}\|\Omega\|_{L^{s}\left(S^{n-1}\right)}\left\|f_{j}\right\|_{L^{q_{1}(\cdot)}\left(\mathbb{R}^{n}\right)}
\end{aligned}
$$

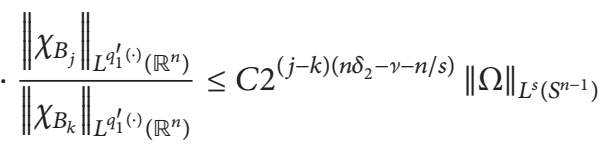

$$
\begin{aligned}
& \cdot\left\|f_{j}\right\|_{L^{q_{1}(\cdot)}\left(\mathbb{R}^{n}\right)} \cdot
\end{aligned}
$$

So we have

$$
\begin{aligned}
I_{1}= & C \sup _{L \in \mathbb{Z}} 2^{-L \lambda p_{1}} \\
& \cdot \sum_{k=-\infty}^{L} 2^{k \alpha p_{1}}\left(\sum_{j=-\infty}^{k-2}\left\|T_{\Omega, \sigma}\left(f_{j}\right) \chi_{k}\right\|_{L^{q_{2}(\cdot)\left(\mathbb{R}^{n}\right)}}\right)^{p_{1}} \\
& \leq C\|\Omega\|_{L^{s}\left(S^{n-1}\right)}^{p_{1}} \sup _{L \in \mathbb{Z}} 2^{-L \lambda p_{1}} \\
& \cdot \sum_{k=-\infty}^{L} 2^{k \alpha p_{1}}\left(\sum_{j=-\infty}^{k-2} 2^{(j-k)\left(n \delta_{2}-\nu-n / s\right)}\left\|f_{j}\right\|_{L^{q_{1}(\cdot)}\left(\mathbb{R}^{n}\right)}\right)^{p_{1}} .
\end{aligned}
$$


When $1<p_{1}<\infty$, take $1 / p_{1}+1 / p_{1}^{\prime}=1$. Since $n \delta_{2}-v-n / s-$ $\alpha>0$, by the Hölder inequality, we have

$$
\begin{aligned}
& I_{1} \leq C\|\Omega\|_{L^{s}\left(S^{n-1}\right)}^{p_{1}} \sup _{L \in \mathbb{Z}} 2^{-L \lambda p_{1}} \\
& \cdot \sum_{k=-\infty}^{L}\left(\sum_{j=-\infty}^{k-2} 2^{j \alpha p_{1}} 2^{(j-k)\left(n \delta_{2}-v-n / s-\alpha\right) p_{1} / 2}\left\|f_{j}\right\|_{L^{q_{1}(\cdot)}\left(\mathbb{R}^{n}\right)}^{p_{1}}\right) \\
& \times\left(\sum_{j=-\infty}^{k-2} 2^{(j-k)\left(n \delta_{2}-v-n / s-\alpha\right) p_{1}^{\prime} / 2}\right)^{p_{1} / p_{1}^{\prime}} \leq C\|\Omega\|_{L^{s}\left(S^{n-1}\right)}^{p_{1}} \\
& \cdot \sup _{L \in \mathbb{Z}} 2^{-L \lambda p_{1}} \\
& \cdot \sum_{k=-\infty}^{L} \sum_{j=-\infty}^{k-2} 2^{j \alpha p_{1}} 2^{(j-k)\left(n \delta_{2}-v-n / s-\alpha\right) p_{1} / 2}\left\|f_{j}\right\|_{L^{q_{1} \cdot(\cdot)}\left(\mathbb{R}^{n}\right)}^{p_{1}} \\
& \leq C\|\Omega\|_{L^{s}\left(S^{n-1}\right)}^{p_{1}} \sup _{L \in \mathbb{Z}} 2^{-L \lambda p_{1}} \sum_{j=-\infty}^{L-2} 2^{j \alpha p_{1}}\left\|f_{j}\right\|_{L^{q_{1}(\cdot)}\left(\mathbb{R}^{n}\right)}^{p_{1}} \\
& \cdot \sum_{k=j+2}^{L} 2^{(j-k)\left(n \delta_{2}-v-n / s-\alpha\right) p_{1} / 2} \leq C\|\Omega\|_{L^{s}\left(S^{n-1}\right)}^{p_{1}} \sup _{L \in \mathbb{Z}} 2^{-L \lambda p_{1}} \\
& \cdot \sum_{j=-\infty}^{L-2} 2^{j \alpha p_{1}}\left\|f_{j}\right\|_{L^{q_{1} \cdot(\cdot)}\left(\mathbb{R}^{n}\right)}^{p_{1}} \leq C\|\Omega\|_{L^{s}\left(S^{n-1}\right)}^{p_{1}}\|f\|_{M \dot{K}_{q_{1}(\cdot)}^{\alpha, p_{1}}\left(\mathbb{R}^{n}\right)}^{p_{1}} .
\end{aligned}
$$

When $0<p_{1} \leq 1$, we have

$$
\begin{aligned}
I_{1} \leq & C\|\Omega\|_{L^{s}\left(S^{n-1}\right)}^{p_{1}} \sup _{L \in \mathbb{Z}} 2^{-L \lambda p_{1}} \sum_{k=-\infty}^{L} 2^{k \alpha p_{1}} \\
& \cdot \sum_{j=-\infty}^{k-2} 2^{(j-k)\left(n \delta_{2}-v-n / s\right) p_{1}}\left\|f_{j}\right\|_{L^{q_{1} \cdot(\cdot)}\left(\mathbb{R}^{n}\right)}^{p_{1}}=C\|\Omega\|_{L^{s}\left(S^{n-1}\right)}^{p_{1}} \\
& \cdot \sup _{L \in \mathbb{Z}} 2^{-L \lambda p_{1}} \sum_{j=-\infty}^{L-2} 2^{j \alpha p_{1}}\left\|f_{j}\right\|_{L^{q_{1} \cdot \cdot()}\left(\mathbb{R}^{n}\right)}^{p_{1}} \\
& \cdot \sum_{k=j+2}^{L} 2^{(j-k)\left(n \delta_{2}-v-n / s-\alpha\right) p_{1}} \leq C\|\Omega\|_{L^{s}\left(S^{n-1}\right)}^{p_{1}} \sup _{L \in \mathbb{Z}} 2^{-L \lambda p_{1}} \\
& \cdot \sum_{j=-\infty}^{L-2} 2^{j \alpha p_{1}}\left\|f_{j}\right\|_{L^{q_{1}} \cdot(\cdot)\left(\mathbb{R}^{n}\right)}^{p_{1}} \leq C\|\Omega\|_{L^{s}\left(S^{n-1}\right)}^{p_{1}} \\
& \cdot\|f\|_{M \dot{K}_{q_{1}(\cdot)}^{\alpha, p_{1}}\left(\mathbb{R}^{n}\right)}^{p_{1}} \cdot
\end{aligned}
$$

Next we estimate $I_{2}$; by the $\left(L^{q_{1} \cdot(\cdot)}\left(\mathbb{R}^{n}\right), L^{q_{2}(\cdot)}\left(\mathbb{R}^{n}\right)\right)$ boundedness of the commutator $T_{\Omega, \sigma}$ we have

$$
\begin{aligned}
I_{2} & =C \sup _{L \in \mathbb{Z}} 2^{-L \lambda p_{1}} \\
& \cdot \sum_{k=-\infty}^{L} 2^{k \alpha p_{1}}\left(\sum_{j=k-1}^{\infty}\left\|T_{\Omega, \sigma}\left(f_{j}\right) \chi_{k}\right\|_{L^{q_{2} \cdot(\cdot)}\left(\mathbb{R}^{n}\right)}\right)^{p_{1}} \leq C
\end{aligned}
$$

$$
\begin{aligned}
& \cdot \sup _{L \in \mathbb{Z}} 2^{-L \lambda p_{1}} \sum_{k=-\infty}^{L} 2^{k \alpha p_{1}}\left(\sum_{j=k-1}^{\infty}\left\|f_{j}\right\|_{L^{q_{1} \cdot(\cdot)}\left(\mathbb{R}^{n}\right)}\right)^{p_{1}}=C \\
& \cdot \sup _{L \in \mathbb{Z}} 2^{-L \lambda p_{1}} \sum_{k=-\infty}^{L}\left(\sum_{j=k-1}^{\infty} 2^{(k-j) \alpha} 2^{j \alpha}\left\|f_{j}\right\|_{L^{q_{1} \cdot(\cdot)}\left(\mathbb{R}^{n}\right)}\right)^{p_{1}} .
\end{aligned}
$$

If $0<p_{1} \leq 1$, then we have

$$
\begin{aligned}
I_{2} \leq C \sup _{L \in \mathbb{Z}} 2^{-L \lambda p_{1}} \sum_{k=-\infty}^{L} \sum_{j=k-1}^{\infty} 2^{(k-j) \alpha p_{1}} 2^{j \alpha p_{1}}\left\|f_{j}\right\|_{L^{q_{1}(\cdot)}\left(\mathbb{R}^{n}\right)}^{p_{1}} \\
\leq C \sup _{L \in \mathbb{Z}} 2^{-L \lambda p_{1}} \sum_{k=-\infty}^{L} \sum_{j=k-1}^{L-1} 2^{(k-j) \alpha p_{1}} 2^{j \alpha p_{1}}\left\|f_{j}\right\|_{L^{q_{1}(\cdot)}\left(\mathbb{R}^{n}\right)}^{p_{1}} \\
\quad+C \sup _{L \in \mathbb{Z}} 2^{-L \lambda p_{1}} \sum_{k=-\infty}^{L} \sum_{j=L}^{\infty} 2^{(k-j) \alpha p_{1}} 2^{j \alpha p_{1}}\left\|f_{j}\right\|_{L^{q_{1} \cdot(\cdot)\left(\mathbb{R}^{n}\right)}}^{p_{1}} \\
=: I_{21}+I_{22} .
\end{aligned}
$$

For $I_{21}$, we have

$$
\begin{aligned}
I_{21} & \leq C \sup _{L \in \mathbb{Z}} 2^{-L \lambda p_{1}} \sum_{j=-\infty}^{L-1} 2^{j \alpha p_{1}}\left\|f_{j}\right\|_{L^{q_{1} \cdot(\cdot)\left(\mathbb{R}^{n}\right)}}^{p_{1}} \sum_{k=-\infty}^{j+1} 2^{(k-j) \alpha p_{1}} \\
& \leq C \sup _{L \in \mathbb{Z}} 2^{-L \lambda p_{1}} \sum_{j=-\infty}^{L-1} 2^{j \alpha p_{1}}\left\|f_{j}\right\|_{L^{q_{1} \cdot(\cdot)\left(\mathbb{R}^{n}\right)}}^{p_{1}} \\
& \leq C\|f\|_{M \dot{K}_{q_{1} \cdot(\cdot)}^{\alpha, p_{1}}\left(\mathbb{R}^{n}\right)}^{p_{1}}
\end{aligned}
$$

For $I_{22}$, by $0<\lambda<\alpha$ we have

$$
\begin{aligned}
& I_{22}=C \sup _{L \in \mathbb{Z}} 2^{-L \lambda p_{1}} \sum_{k=-\infty}^{L} \sum_{j=L}^{\infty} 2^{(k-j) \alpha p_{1}} 2^{j \alpha p_{1}}\left\|f_{j}\right\|_{L^{q_{1}} \cdot\left(\mathbb{R}^{n}\right)}^{p_{1}} \leq C \\
& \cdot \sup 2^{-L \lambda p_{1}} \\
& \cdot \sum_{k=-\infty}^{L} \sum_{j=L}^{\infty} 2^{(k-j) \alpha p_{1}} 2^{j \lambda p_{1}} 2^{-j \lambda p_{1}}\left(\sum_{m=-\infty}^{j} 2^{m \alpha p_{1}}\left\|f_{m}\right\|_{L^{q_{1}(\cdot)}\left(\mathbb{R}^{n}\right)}^{p_{p_{1}}}\right) \\
& \leq C \sup _{L \in \mathbb{Z}} 2^{-L \lambda p_{1}} \sum_{k=-\infty}^{L} \sum_{j=L}^{\infty} 2^{(k-j) \alpha p_{1}} 2^{j \lambda p_{1}}\|f\|_{M K_{q_{1} \cdot(\cdot)}^{\alpha, R^{n}}}^{p_{1}} \\
& =C\|f\|_{M \dot{K}_{q 1}^{\alpha, \cdot}\left(\mathbb{R}^{n}\right)}^{p_{1}} \sup _{L \in \mathbb{Z}} 2^{-L \lambda p_{1}} \sum_{k=-\infty}^{L} 2^{k \alpha p_{1}} \sum_{j=L}^{\infty} 2^{j(\lambda-\alpha) p_{1}} \\
& \leq C\|f\|_{M \dot{q}_{q_{1}(\cdot)}^{\alpha, p_{1}}\left(\mathbb{R}^{n}\right)}^{p_{L \in \mathbb{Z}}} \sup ^{-L \lambda p_{1}} 2^{L \alpha p_{1}} 2^{L(\lambda-\alpha) p_{1}}
\end{aligned}
$$

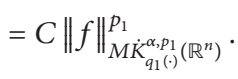


If $1<p_{1}<\infty$, noting $\lambda<\alpha$, we can take a constant $\eta>1$ so that $\lambda-\alpha / \eta<0$. By the Hölder inequality we have

$$
\begin{aligned}
I_{2} \leq & C \sup _{L \in \mathbb{Z}} 2^{-L \lambda p_{1}} \\
& \cdot \sum_{k=-\infty}^{L}\left(\sum_{j=k-1}^{\infty} 2^{(k-j) \alpha p_{1} / \eta} 2^{j \alpha p_{1}}\left\|f_{j}\right\|_{L^{q_{1} \cdot(\cdot)}\left(\mathbb{R}^{n}\right)}^{p_{1}}\right) \\
& \cdot\left(\sum_{j=k-1}^{\infty} 2^{(k-j) \alpha p_{1}^{\prime}(\eta-1) / \eta}\right)^{p_{1} / p_{1}^{\prime}} \leq C \sup _{L \in \mathbb{Z}} 2^{-L \lambda p_{1}} \\
& \cdot \sum_{k=-\infty}^{L} \sum_{j=k-1}^{\infty} 2^{(k-j) \alpha p_{1} / \eta} 2^{j \alpha p_{1}}\left\|f_{j}\right\|_{L^{q_{1}(\cdot)}\left(\mathbb{R}^{n}\right)}^{p_{p}} \leq C \\
& \cdot \sup _{L \in \mathbb{Z}} 2^{-L \lambda p_{1}} \sum_{k=-\infty}^{L} \sum_{j=k-1}^{L-1} 2^{(k-j) \alpha p_{1} / \eta} 2^{j \alpha p_{1}}\left\|f_{j}\right\|_{L^{q_{1} \cdot(\cdot)}\left(\mathbb{R}^{n}\right)}^{p_{1}} \\
& +C \sup _{L \in \mathbb{Z}} 2^{-L \lambda p_{1}} \sum_{k=-\infty}^{L} \sum_{j=L}^{\infty} 2^{(k-j) \alpha p_{1} / \eta} 2^{j \alpha p_{1}}\left\|f_{j}\right\|_{L^{q_{1} \cdot(\cdot)}\left(\mathbb{R}^{n}\right)}^{p_{1}} \\
& =I_{23}+I_{24} \cdot
\end{aligned}
$$

For $I_{23}$, we have

$$
\begin{aligned}
& I_{23} \\
& \quad \leq C \sup _{L \in \mathbb{Z}} 2^{-L \lambda p_{1}} \sum_{j=-\infty}^{L-1} 2^{j \alpha p_{1}}\left\|f_{j}\right\|_{L^{q_{1} \cdot(\cdot)}}^{p_{1^{n}}} \sum_{k=-\infty}^{j+1} 2^{(k-j) \alpha p_{1} / \eta} \\
& \quad \leq C \sup _{L \in \mathbb{Z}} 2^{-L \lambda p_{1}} \sum_{j=-\infty}^{L-1} 2^{j \alpha p_{1}}\left\|f_{j}\right\|_{L^{q_{1} \cdot(\cdot)}\left(\mathbb{R}^{n}\right)}^{p_{1}} \\
& \left.\leq C\|f\|_{M \dot{K}_{q_{1}(\cdot)}^{\alpha, p_{1}}}^{p_{1}} \mathbb{R}^{n}\right)
\end{aligned}
$$

For $I_{24}$, by $0<\lambda<\alpha / \eta$ we have

$$
\begin{aligned}
& I_{24}=C \sup _{L \in \mathbb{Z}} 2^{-L \lambda p_{1}} \sum_{k=-\infty}^{L} \sum_{j=L}^{\infty} 2^{(k-j) \alpha p_{1} / \eta} 2^{j \alpha p_{1}}\left\|f_{j}\right\|_{L^{q_{1}(\cdot)}\left(\mathbb{R}^{n}\right)}^{p_{1}} \\
& \leq C \sup _{L \in \mathbb{Z}} 2^{-L \lambda p_{1}} \sum_{k=-\infty}^{L} \sum_{j=L}^{\infty} 2^{(k-j) \alpha p_{1} / \eta} 2^{j \lambda p_{1}}\|f\|_{M \dot{K}_{q_{1}(\cdot)}^{\alpha, p_{1}}\left(\mathbb{R}^{n}\right)}^{p_{1}} \\
& =C\|f\|_{M \dot{K}_{q_{1}(\cdot)}^{\alpha, p_{1}\left(\mathbb{R}^{n}\right)}}^{p_{1}} \sup _{L \in \mathbb{Z}} 2^{-L \lambda p_{1}} \sum_{k=-\infty}^{L} 2^{k \alpha p_{1} / \eta} \sum_{j=L}^{\infty} 2^{j(\lambda-\alpha / \eta) p_{1}} \\
& \leq C\|f\|_{M \dot{K}_{q_{1}(\cdot)}^{\alpha, p_{1}}}^{p_{1}} \sup _{L \in \mathbb{Z}} 2^{-L \lambda p_{1}} 2^{L \alpha p_{1} / \eta} 2^{L(\lambda-\alpha / \eta) p_{1}} \\
& =C\|f\|_{M \dot{K}_{q_{1}(\cdot)}^{\alpha, p_{1}}\left(\mathbb{R}^{n}\right)}^{p_{1}} \text {. }
\end{aligned}
$$

Thus, by (28), (37), and (39)-(44) we complete the proof of Theorem 11.

Remark 15. In [22], Abdalmonem et al. considered the boundedness of fractional integral with variable kernel on variable exponent Herz-Morrey spaces (see Theorem A, pp. 790). It is different from Theorem 11. Theorem 11 is under the weaker conditions than Theorem A. For example, the conditions of Theorem A are $\Omega \in L^{r}\left(S^{n-1}\right)\left(r>p_{2}^{+}\right)$ and satisfy $\int_{0}^{1} \omega_{r}(\delta) / \delta^{1+\beta} d \delta<\infty$. But we do not need the condition $\int_{0}^{1} \omega_{r}(\delta) / \delta^{1+\beta} d \delta<\infty$ in Theorem 11.

\section{BMO Estimate for the Commutator of Homogeneous Fractional Integral Operator}

Let us first recall that the space $\operatorname{BMO}\left(\mathbb{R}^{n}\right)$ consists of all locally integrable functions $f$ such that

$$
\|f\|_{*}=\sup _{\mathrm{Q}} \frac{1}{|Q|} \int_{\mathrm{Q}}\left|f(x)-f_{\mathrm{Q}}\right| d x<\infty,
$$

where $f_{\mathrm{Q}}=|\mathrm{Q}|^{-1} \int_{\mathrm{Q}} f(y) d y$, the supremum is taken over all cubes $Q \subset \mathbb{R}^{n}$ with sides parallel to the coordinate axes, and $|Q|$ denotes the Lebesgue measure of $Q$.

A nonnegative locally integrable function $\omega(x)$ on $\mathbb{R}^{n}$ is said to belong to $A(p, q)(1<p, q<\infty)$, if there is a constant $C>0$ such that

$$
\begin{aligned}
& \sup _{Q}\left(\frac{1}{|Q|} \int_{Q} \omega(x)^{q} d x\right)^{1 / q}\left(\frac{1}{|Q|} \int_{Q} \omega(x)^{-p^{\prime}} d x\right)^{1 / p^{\prime}} \\
& \quad \leq C<\infty,
\end{aligned}
$$

where $p^{\prime}=p /(p-1)$.

Let $b \in \mathrm{BMO}\left(\mathbb{R}^{n}\right)$. The weighted $\left(L^{p}, L^{q}\right)$ boundedness of $\left[b, T_{\Omega, \sigma}\right]$ has been proved by Segovia and Torrea [23], Ding [24], and Ding and Lu [25], respectively.

Lemma 16 (see [24]). Suppose that $0<\sigma<n, s^{\prime}<p<n / \sigma$, $1 / q=1 / p-\sigma / n, \Omega \in L^{s}\left(S^{n-1}\right)$, and $\omega^{s^{\prime}} \in A\left(p / s^{\prime}, q / s^{\prime}\right)$, then for $b \in B M O\left(\mathbb{R}^{n}\right), m \in \mathbb{Z}$, there is a constant $C$, independent of $f$, such that

$$
\begin{aligned}
& \left(\int_{\mathbb{R}^{n}}\left|\left[b, T_{\Omega, \sigma}\right]^{m} f(x) \omega(x)\right|^{q} d x\right)^{1 / q} \\
& \quad \leq C\left(\int_{\mathbb{R}^{n}}|f(x) \omega(x)|^{p} d x\right)^{1 / p} .
\end{aligned}
$$

We say that $\omega \in A_{1}$ if $M \omega(x) \leq C \omega(x)$ for a.e. $x$.

Lemma 17 (see [26]). Given a family $\mathscr{F}$ and an open set $E \subset$ $\mathbb{R}^{n}$, assume that, for some $p_{0}$ and $q_{0}, 0<p_{0} \leq q_{0}<\infty$, and every weight $\omega \in A_{1}$

$$
\begin{aligned}
& \left(\int_{E} f(x)^{q_{0}} \omega(x) d x\right)^{1 / q_{0}} \\
& \quad \leq C_{0}\left(\int_{E} g(x)^{p_{0}} \omega(x)^{p_{0} / q_{0}} d x\right)^{1 / p_{0}}, \quad(f, g) \in \mathscr{F} .
\end{aligned}
$$

Given $p(\cdot) \in \mathscr{P}^{0}(E)$ such that $p_{0}<p_{-} \leq p_{+}<p_{0} q_{0} /\left(q_{0}-p_{0}\right)$, define the function $q(\cdot)$ by

$$
\frac{1}{p(x)}-\frac{1}{q(x)}=\frac{1}{p_{0}}-\frac{1}{q_{0}}, \quad x \in E .
$$


If $p(\cdot)$ satisfies (8) in Lemma 1 , then for all $(f, g) \in \mathscr{F}$ such that $f \in L^{q(\cdot)}(E)$

$$
\|f\|_{L^{q(\cdot)}(E)} \leq C\|g\|_{L^{p(\cdot)}(E)} .
$$

Similar to the method of [16, Theorem 1.9], by Lemmas 16 and 17 , it is easy to get the $\left(L^{p(\cdot)}\left(\mathbb{R}^{n}\right), L^{q(\cdot)}\left(\mathbb{R}^{n}\right)\right)$-boundedness of the commutator $\left[b, T_{\Omega, \sigma}\right]$.

Next, we will give the corresponding result about the commutator $\left[b, T_{\Omega, \sigma}\right]$ on Morrey-Herz spaces with variable exponent.

Theorem 18. Suppose that $b \in B M O\left(\mathbb{R}^{n}\right), 0<v \leq 1,0<\sigma<$ $n-v, q_{1}(\cdot) \in \mathscr{P}\left(\mathbb{R}^{n}\right)$ satisfy conditions (8) in Lemma 1 with $q_{1}^{+}<n / \sigma, 1 / q_{1}(x)-1 / q_{2}(x)=\sigma / n$, and $\Omega \in L^{s}\left(S^{n-1}\right)(s>$ $\left.q_{1}^{\prime-}\right)$. Let $0<p_{1} \leq p_{2}<\infty$ and $0<\lambda<\alpha<n \delta_{2}-\nu-n / s$ (or $0<\lambda<\alpha_{2} \leq \alpha_{1}<n \delta_{2}-v-n / s$ ). Then $\left[b, T_{\Omega, \sigma}\right]$ is bounded from $M \dot{K}_{q_{1} \cdot(\cdot)}^{\alpha, p_{1}}\left(\mathbb{R}^{n}\right)$ (or $\left.M K_{q_{1}(\cdot)}^{\alpha_{1}, p_{1}}\left(\mathbb{R}^{n}\right)\right)$ to $M \dot{K}_{q_{2}(\cdot)}^{\alpha, p_{2}}\left(\mathbb{R}^{n}\right)$ $\left(\right.$ or $\left.M K_{q_{2}(\cdot)}^{\alpha_{2}, p_{2}}\left(\mathbb{R}^{n}\right)\right)$.

In the proof of Theorem 18, we also need the following lemmas.

Lemma 19 (see [27]). Let $p(\cdot) \in \mathscr{B}\left(\mathbb{R}^{n}\right), k$ be a positive integer, and $B$ be a ball in $\mathbb{R}^{n}$. Then we have that for all $b \in$ $B M O\left(\mathbb{R}^{n}\right)$ and all $j, i \in \mathbb{Z}$ with $j>i$

$$
\begin{aligned}
& \frac{1}{C}\|b\|_{*}^{k} \leq \sup _{B} \frac{1}{\left\|\chi_{B}\right\|_{L^{(\cdot)}\left(\mathbb{R}^{n}\right)}}\left\|\left(b-b_{B}\right)^{k} \chi_{B}\right\|_{L^{p(\cdot)}\left(\mathbb{R}^{n}\right)} \\
& \quad \leq C\|b\|_{*}^{k}, \\
& \left\|\left(b-b_{B_{i}}\right)^{k} \chi_{B_{j}}\right\|_{L^{p(\cdot)}\left(\mathbb{R}^{n}\right)} \leq C(j-i)^{k}\|b\|_{*}^{k}\left\|\chi_{B_{j}}\right\|_{L^{(\cdot)}\left(\mathbb{R}^{n}\right)},
\end{aligned}
$$

where $B_{i}=\left\{x \in \mathbb{R}^{n}:|x| \leq 2^{i}\right\}$ and $B_{j}=\left\{x \in \mathbb{R}^{n}:|x| \leq 2^{j}\right\}$.

Proof of Theorem 18. We only prove the homogeneous case. The nonhomogeneous case can be proved in the same way. Let $f \in M \dot{K}_{q_{1}(\cdot)}^{\alpha, p_{1}}\left(\mathbb{R}^{n}\right)$ and $b \in \operatorname{BMO}\left(\mathbb{R}^{n}\right)$. Denote $f_{j}=f \chi_{j}$ for each $j \in \mathbb{Z}$; then we have $f(x)=\sum_{j=-\infty}^{\infty} f_{j}(x)$. Note that $p_{1} \leq p_{2}$; we have

$$
\begin{aligned}
& \left\|\left[b, T_{\Omega, \sigma}\right](f)\right\|_{\left.M \dot{K}_{q_{2}, \cdot()^{\prime}}^{\alpha, p_{2}} \mathbb{R}^{n}\right)}^{p_{1}} \\
& =\sup _{L \in \mathbb{Z}} 2^{-L \lambda p_{1}}\left\{\sum_{k=-\infty}^{L} 2^{k \alpha p_{2}}\left\|\left[b, T_{\Omega, \sigma}\right](f) \chi_{k}\right\|_{L^{2 q_{2}(\cdot)}\left(\mathbb{R}^{n}\right)}^{p_{2}}\right\}^{p_{1} / p_{2}} \\
& \leq \sup _{L \in \mathbb{Z}} 2^{-L \lambda p_{1}} \sum_{k=-\infty}^{L} 2^{k \alpha p_{1}}\left\|\left[b, T_{\Omega, \sigma}\right](f) \chi_{k}\right\|_{L^{q_{2}(\cdot)}\left(\mathbb{R}^{n}\right)}^{p_{1}} \\
& \leq C \sup _{L \in \mathbb{Z}} 2^{-L \lambda p_{1}} \sum_{k=-\infty}^{L} 2^{k \alpha p_{1}}\left(\sum_{j=-\infty}^{k-2}\left\|\left[b, T_{\Omega, \sigma}\right]\left(f_{j}\right) \chi_{k}\right\|_{L_{2}(\cdot)\left(\mathbb{R}^{n}\right)}\right)^{p_{1}} \\
& +C \sup _{L \in \mathbb{Z}} 2^{-L \lambda p_{1}} \sum_{k=-\infty}^{L} 2^{k \alpha p_{1}}\left(\sum_{j=k-1}^{\infty}\left\|\left[b, T_{\Omega, \sigma}\right]\left(f_{j}\right) \chi_{k}\right\|_{L^{q_{2}} \cdot(\cdot)\left(\mathbb{R}^{n}\right)}\right)^{p_{1}} \\
& =: J_{1}+J_{2} \text {. }
\end{aligned}
$$

We first estimate $J_{1}$. For each $k \in \mathbb{Z}, j \leq k-2$, and a.e. $x \in A_{k}$, using the generalized Hölder inequality we have

$$
\begin{aligned}
& \left|\left[b, T_{\Omega, \sigma}\right]\left(f_{j}\right)(x)\right| \leq C \int_{B_{j}} \frac{|\Omega(x-y)|}{|x-y|^{n-\sigma}}|b(x)-b(y)| \\
& \cdot\left|f_{j}(y)\right| d y \leq C 2^{k(\sigma-n)} \int_{B_{j}}|\Omega(x-y)| \\
& \cdot|b(x)-b(y)|\left|f_{j}(y)\right| d y \\
& \quad \leq C 2^{k(\sigma-n)}\left(\left|b(x)-b_{B_{j}}\right| \int_{B_{j}}|\Omega(x-y)|\left|f_{j}(y)\right| d y\right. \\
& \left.\quad+\int_{B_{j}}|\Omega(x-y)|\left|b_{B_{j}}-b(y)\right|\left|f_{j}(y)\right| d y\right) \\
& \quad \leq C 2^{k(\sigma-n)}\left(\left|b(x)-b_{B_{j}}\right|\left\|\Omega(x-\cdot) \chi_{j}(\cdot)\right\|_{L^{q_{1}^{\prime}(\cdot)}\left(\mathbb{R}^{n}\right)}\right. \\
& \cdot\left\|f_{j}\right\|_{L^{q_{1}(\cdot)}\left(\mathbb{R}^{n}\right)} \\
& \quad+\left\|\Omega(x-\cdot)\left(b_{B_{j}}-b(\cdot)\right) \chi_{j}(\cdot)\right\|_{L^{q_{1}^{\prime} \cdot(\cdot)}\left(\mathbb{R}^{n}\right)} \\
& \left.\quad \cdot\left\|f_{j}\right\|_{L^{q_{1}(\cdot)}\left(\mathbb{R}^{n}\right)}\right) .
\end{aligned}
$$

Noting $s>q_{1}^{\prime-}$, we denote $\widetilde{q_{1}^{\prime}}(\cdot)>1$ and $1 / q_{1}^{\prime}(x)=$ $1 / \widetilde{q_{1}^{\prime}}(x)+1 / s$. By Lemmas 12 and 13 we have

$$
\begin{aligned}
& \left\|\Omega(x-\cdot) \chi_{j}(\cdot)\right\|_{L^{q_{1}^{\prime} \cdot(\cdot)\left(\mathbb{R}^{n}\right)}} \\
& \leq\left\|\Omega(x-\cdot) \chi_{j}(\cdot)\right\|_{L^{s}\left(\mathbb{R}^{n}\right)}\left\|\chi_{j}(\cdot)\right\|_{L^{\tau_{1}^{\top}(\cdot)}\left(\mathbb{R}^{n}\right)} \\
& \leq\left\|\Omega(x-\cdot) \chi_{j}(\cdot)\right\|_{L^{s}\left(\mathbb{R}^{n}\right)}\left\|\chi_{B_{j}}\right\|_{L^{\tau_{1}} \cdot(\cdot)\left(\mathbb{R}^{n}\right)} \\
& \leq C 2^{-j v}\left(\int_{A_{j}}|\Omega(x-y)|^{s}|y|^{s v} d y\right)^{1 / s}\left\|\chi_{B_{j}}\right\|_{L^{\tau_{1}}(\cdot)\left(\mathbb{R}^{n}\right)} \\
& \leq C 2^{-j v} 2^{k(v+n / s)}\|\Omega\|_{L^{s}\left(S^{n-1}\right)}\left\|\chi_{B_{j}}\right\|_{L^{\tau_{1}^{(} \cdot(\cdot)}\left(\mathbb{R}^{n}\right)} .
\end{aligned}
$$

When $\left|B_{j}\right| \leq 2^{n}$ and $x_{j} \in B_{j}$, by Lemma 14 we have

$$
\left\|\chi_{B_{j}}\right\|_{L^{q_{1}^{\prime}(\cdot)}\left(\mathbb{R}^{n}\right)} \approx\left|B_{j}\right|^{1 \widetilde{q_{1}^{\prime}(}\left(x_{j}\right)} \approx\left\|\chi_{B_{j}}\right\|_{L_{1_{1}^{\prime}(\cdot)}^{q^{\prime}\left(\mathbb{R}^{n}\right)}}\left|B_{j}\right|^{-1 / s} .
$$

When $\left|B_{j}\right| \geq 1$ we have

$$
\left\|\chi_{B_{j}}\right\|_{L^{\bar{q}_{1}^{\top} \cdot(\cdot)\left(\mathbb{R}^{n}\right)}} \approx\left|B_{j}\right|^{1 / q_{1}^{\prime}(\infty)} \approx\left\|\chi_{B_{j}}\right\|_{L_{1_{1}^{\prime}(\cdot)\left(\mathbb{R}^{n}\right)}}\left|B_{j}\right|^{-1 / s} .
$$

So we obtain $\left\|\chi_{B_{j}}\right\|_{L^{q_{1}^{\top} \cdot(\cdot)}\left(\mathbb{R}^{n}\right)} \approx\left\|\chi_{B_{j}}\right\|_{L^{q_{1}^{\prime} \cdot(\cdot)\left(\mathbb{R}^{n}\right)}}\left|B_{j}\right|^{-1 / s}$.

So we have

$$
\begin{aligned}
& \left\|\Omega(x-\cdot) \chi_{j}(\cdot)\right\|_{L^{q_{1}^{\prime}(\cdot)}\left(\mathbb{R}^{n}\right)} \\
& \quad \leq C 2^{(k-j)(v+n / s)}\|\Omega\|_{L^{s}\left(S^{n-1}\right)}\left\|\chi_{B_{j}}\right\|_{L^{q_{1}^{\prime}(\cdot)}\left(\mathbb{R}^{n}\right)} .
\end{aligned}
$$


8

Journal of Function Spaces

Similarly, by Lemma 19 we have

$$
\begin{aligned}
& \left\|\Omega(x-\cdot)\left(b_{B_{j}}-b(\cdot)\right) \chi_{j}(\cdot)\right\|_{L^{q_{1}^{\prime}(\cdot)}\left(\mathbb{R}^{n}\right)} \\
& \quad \leq\left\|\Omega(x-\cdot) \chi_{j}(\cdot)\right\|_{L^{s}\left(\mathbb{R}^{n}\right)}\left\|\left(b_{B_{j}}-b(\cdot)\right) \chi_{j}(\cdot)\right\|_{L^{q_{1}^{\top}(\cdot)}\left(\mathbb{R}^{n}\right)} \\
& \quad \leq C\|b\|_{*}\left\|\chi_{B_{j}}\right\|_{L^{q_{1}^{\prime}(\cdot)}\left(\mathbb{R}^{n}\right)}\left\|\Omega(x-\cdot) \chi_{j}(\cdot)\right\|_{L^{s}\left(\mathbb{R}^{n}\right)} \\
& \quad \leq C\|b\|_{*} 2^{(k-j)(\nu+n / s)}\|\Omega\|_{L^{s}\left(S^{n-1}\right)}\left\|\chi_{B_{j}}\right\|_{L^{q_{1}^{\prime}(\cdot)\left(\mathbb{R}^{n}\right)}} .
\end{aligned}
$$

By (57), (58), and Lemmas 3, 4, and 19 we have

$$
\begin{aligned}
& \left\|\left[b, T_{\Omega, \sigma}\right]\left(f_{j}\right) \chi_{k}\right\|_{L^{q_{2}(\cdot)}\left(\mathbb{R}^{n}\right)} \\
& \leq C 2^{k(\sigma-n)}\left(2^{(k-j)(v+n / s)}\|\Omega\|_{L^{s}\left(S^{n-1}\right)}\left\|\chi_{B_{j}}\right\|_{L^{q_{1}^{\prime}(\cdot)}\left(\mathbb{R}^{n}\right)}\right. \\
& \cdot\left\|\left(b(\cdot)-b_{B_{j}}\right) \chi_{k}(\cdot)\right\|_{L^{q_{2}(\cdot)\left(\mathbb{R}^{n}\right)}}\left\|f_{j}\right\|_{L^{q_{1}(\cdot)\left(\mathbb{R}^{n}\right)}}+\|b\|_{*} \\
& \cdot 2^{(k-j)(\nu+n / s)}\|\Omega\|_{L^{s}\left(S^{n-1}\right)}\left\|\chi_{B_{j}}\right\|_{L^{q_{1}^{\prime}(\cdot)}\left(\mathbb{R}^{n}\right)}\left\|\chi_{k}\right\|_{L^{q_{2}(\cdot)}\left(\mathbb{R}^{n}\right)} \\
& \left.\cdot\left\|f_{j}\right\|_{L^{q_{1}(\cdot)}\left(\mathbb{R}^{n}\right)}\right) \leq C 2^{k(\sigma-n)}\left((k-j)\|b\|_{*}\right. \\
& \cdot 2^{(k-j)(v+n / s)}\|\Omega\|_{L^{s}\left(S^{n-1}\right)}\left\|\chi_{B_{j}}\right\|_{L^{q_{1}^{\prime}(\cdot)}\left(\mathbb{R}^{n}\right)}\left\|\chi_{B_{k}}\right\|_{L^{q_{2}(\cdot)}\left(\mathbb{R}^{n}\right)} \\
& \cdot\left\|f_{j}\right\|_{L^{q_{1}(\cdot)}\left(\mathbb{R}^{n}\right)}+\|b\|_{*} 2^{(k-j)(v+n / s)}\|\Omega\|_{L^{s}\left(S^{n-1}\right)} \\
& \left.\cdot\left\|\chi_{B_{j}}\right\|_{L^{q_{1}^{\prime} \cdot(\cdot)}\left(\mathbb{R}^{n}\right)}\left\|\chi_{B_{k}}\right\|_{L^{q_{2}(\cdot)}\left(\mathbb{R}^{n}\right)}\left\|f_{j}\right\|_{L^{q_{1}(\cdot)}\left(\mathbb{R}^{n}\right)}\right) \leq C(k \\
& -j)\|b\|_{*} 2^{k(\sigma-n)} 2^{(k-j)(v+n / s)}\|\Omega\|_{L^{s}\left(S^{n-1}\right)}\left\|\chi_{B_{j}}\right\|_{L^{q_{1}^{\prime}(\cdot)}\left(\mathbb{R}^{n}\right)} \\
& \cdot\left\|\chi_{B_{k}}\right\|_{L^{q_{2}(\cdot)}\left(\mathbb{R}^{n}\right)}\left\|f_{j}\right\|_{L^{q_{1}(\cdot)}\left(\mathbb{R}^{n}\right)} \leq C(k-j)\|b\|_{*} \\
& \cdot 2^{-k n} 2^{(k-j)(\nu+n / s)}\|\Omega\|_{L^{s}\left(S^{n-1}\right)}\left\|\chi_{B_{j}}\right\|_{L^{q_{1}^{\prime} \cdot(\cdot)}\left(\mathbb{R}^{n}\right)} \\
& \cdot\left\|T_{\sigma}\left(\chi_{B_{k}}\right)\right\|_{L^{q_{2}(\cdot)}\left(\mathbb{R}^{n}\right)}\left\|f_{j}\right\|_{L^{q_{1}(\cdot)}\left(\mathbb{R}^{n}\right)} \leq C(k-j)\|b\|_{*} \\
& \cdot 2^{-k n} 2^{(k-j)(v+n / s)}\|\Omega\|_{L^{s}\left(S^{n-1}\right)}\left\|f_{j}\right\|_{L^{q_{1}(\cdot)}\left(\mathbb{R}^{n}\right)} \\
& \cdot\left\|\chi_{B_{j}}\right\|_{L^{q_{1}^{\prime}(\cdot)}\left(\mathbb{R}^{n}\right)}\left\|\chi_{B_{k}}\right\|_{L^{q_{1}(\cdot)}\left(\mathbb{R}^{n}\right)} \leq C(k-j)\|b\|_{*} \\
& \cdot 2^{(k-j)(v+n / s)}\|\Omega\|_{L^{s}\left(S^{n-1}\right)}\left\|f_{j}\right\|_{L^{q_{1}(\cdot)}\left(\mathbb{R}^{n}\right)} \frac{\left\|\chi_{B_{j}}\right\|_{L^{q_{1}^{\prime}(\cdot)}\left(\mathbb{R}^{n}\right)}}{\left\|\chi_{B_{k}}\right\|_{L^{q_{1}^{\prime} \cdot(\cdot)}\left(\mathbb{R}^{n}\right)}} \\
& \leq C\|b\|_{*}(k-j) 2^{(j-k)\left(n \delta_{2}-v-n / s\right)}\|\Omega\|_{L^{s}\left(S^{n-1}\right)} \\
& \cdot\left\|f_{j}\right\|_{L^{q_{1}(\cdot)}\left(\mathbb{R}^{n}\right)} .
\end{aligned}
$$

So we have

$$
\begin{aligned}
J_{1}= & C \sup _{L \in \mathbb{Z}} 2^{-L \lambda p_{1}} \\
& \cdot \sum_{k=-\infty}^{L} 2^{k \alpha p_{1}}\left(\sum_{j=-\infty}^{k-2}\left\|\left[b, T_{\Omega, \sigma}\right]\left(f_{j}\right) \chi_{k}\right\|_{L^{q_{2}(\cdot)}\left(\mathbb{R}^{n}\right)}\right)^{p_{1}} \\
& \leq C\|b\|_{*}^{p_{1}}\|\Omega\|_{L^{s}\left(S^{n-1}\right)}^{p_{1}} \sup _{L \in \mathbb{Z}} 2^{-L \lambda p_{1}} \\
& \cdot \sum_{k=-\infty}^{L} 2^{k \alpha p_{1}}\left(\sum_{j=-\infty}^{k-2}(k-j)\right. \\
& \left.\cdot 2^{(j-k)\left(n \delta_{2}-v-n / s\right)}\left\|f_{j}\right\|_{L^{q_{1}(\cdot)}\left(\mathbb{R}^{n}\right)}\right)^{p_{1}} .
\end{aligned}
$$

When $1<p_{1}<\infty$, take $1 / p_{1}+1 / p_{1}^{\prime}=1$. Since $n \delta_{2}-v-n / s-$ $\alpha>0$, by the Hölder inequality, we have

$$
\begin{aligned}
& J_{1} \leq C\|b\|_{*}^{p_{1}}\|\Omega\|_{L^{s}\left(S^{n-1}\right)}^{p_{1}} \sup _{L \in \mathbb{Z}} 2^{-L \lambda p_{1}} \\
& \cdot \sum_{k=-\infty}^{L}\left(\sum_{j=-\infty}^{k-2} 2^{j \alpha p_{1}} 2^{(j-k)\left(n \delta_{2}-\gamma-n / s-\alpha\right) p_{1} / 2}\left\|f_{j}\right\|_{L^{q_{1}(\cdot)}\left(\mathbb{R}^{n}\right)}^{p_{1}}\right) \\
& \times\left(\sum_{j=-\infty}^{k-2} 2^{(j-k)\left(n \delta_{2}-v-n / s-\alpha\right) p_{1}^{\prime} / 2}(k-j)^{p_{1}^{\prime}}\right)^{p_{1} / p_{1}^{\prime}} \\
& \leq C\|b\|_{*}^{p_{1}}\|\Omega\|_{L^{s}\left(S^{n-1}\right)}^{p_{1}} \sup _{L \in \mathbb{Z}} 2^{-L \lambda p_{1}} \\
& \cdot \sum_{k=-\infty}^{L} \sum_{j=-\infty}^{k-2} 2^{j \alpha p_{1}} 2^{(j-k)\left(n \delta_{2}-\gamma-n / s-\alpha\right) p_{1} / 2}\left\|f_{j}\right\|_{L^{q_{1} \cdot(\cdot)}\left(\mathbb{R}^{n}\right)}^{p_{1}} \\
& \leq C\|b\|_{*}^{p_{1}}\|\Omega\|_{L^{s}\left(S^{n-1}\right)}^{p_{1}} \sup _{L \in \mathbb{Z}} 2^{-L \lambda p_{1}} \\
& \cdot \sum_{j=-\infty}^{L-2} 2^{j \alpha p_{1}}\left\|f_{j}\right\|_{L^{q_{1}} \cdot(\cdot)\left(\mathbb{R}^{n}\right)}^{p_{1}} \sum_{k=j+2}^{L} 2^{(j-k)\left(n \delta_{2}-v-n / s-\alpha\right) p_{1} / 2} \\
& \leq C\|b\|_{*}^{p_{1}}\|\Omega\|_{L^{s}\left(S^{n-1}\right)}^{p_{1}} \sup _{L \in \mathbb{Z}} 2^{-L \lambda p_{1}} \sum_{j=-\infty}^{L-2} 2^{j \alpha p_{1}}\left\|f_{j}\right\|_{L^{q_{1} \cdot(\cdot)}\left(\mathbb{R}^{n}\right)}^{p_{1}} \\
& \leq C\|b\|_{*}^{p_{1}}\|\Omega\|_{L^{s}\left(S^{n-1}\right)}^{p_{1}}\|f\|_{M \dot{K}_{q_{1}(\cdot)}^{\alpha, p_{1}}\left(\mathbb{R}^{n}\right)}^{p_{1}} .
\end{aligned}
$$

When $0<p_{1} \leq 1$, we have

$$
\begin{aligned}
J_{1} \leq & C\|b\|_{*}^{p_{1}}\|\Omega\|_{L^{s}\left(S^{n-1}\right)}^{p_{1}} \sup _{L \in \mathbb{Z}} 2^{-L \lambda p_{1}} \\
& \cdot \sum_{k=-\infty}^{L} 2^{k \alpha p_{1}} \sum_{j=-\infty}^{k-2} 2^{(j-k)\left(n \delta_{2}-\gamma-n / s\right) p_{1}}(k-j)^{p_{1}}
\end{aligned}
$$




$$
\begin{aligned}
& \cdot\left\|f_{j}\right\|_{L^{q_{1}(\cdot)\left(\mathbb{R}^{n}\right)}}^{p_{1}}=C\|b\|_{*}^{p_{1}}\|\Omega\|_{L^{s}\left(S^{n-1}\right)}^{p_{1}} \sup _{L \in \mathbb{Z}} 2^{-L \lambda p_{1}} \\
& \cdot \sum_{j=-\infty}^{L-2} 2^{j \alpha p_{1}}\left\|f_{j}\right\|_{L^{q_{1} \cdot(\cdot)}\left(\mathbb{R}^{n}\right)}^{p_{1}} \\
& \cdot \sum_{k=j+2}^{L} 2^{(j-k)\left(n \delta_{2}-v-n / s-\alpha\right) p_{1}}(k-j)^{p_{1}} \leq C\|b\|_{*}^{p_{1}} \\
& \cdot\|\Omega\|_{L^{5}\left(S^{n-1}\right)}^{p_{1}} \sup _{L \in \mathbb{Z}} 2^{-L \lambda p_{1}} \sum_{j=-\infty}^{L-2} 2^{j \alpha p_{1}}\left\|f_{j}\right\|_{L^{q_{1}(\cdot)}\left(\mathbb{R}^{n}\right)}^{p_{1}} \\
& \leq C\|b\|_{*}^{p_{1}}\|\Omega\|_{L^{s}\left(S^{n-1}\right)}^{p_{1}}\|f\|_{M K_{q_{1}(\cdot)}^{\alpha, p_{1}\left(\mathbb{R}^{n}\right)}}^{p_{1}} \cdot
\end{aligned}
$$

Next we estimate $J_{2}$; by the $\left(L^{q_{1}(\cdot)}\left(\mathbb{R}^{n}\right), L^{q_{2}(\cdot)}\left(\mathbb{R}^{n}\right)\right)$ boundedness of the commutator $\left[b, T_{\Omega, \sigma}\right]$ we have

$$
\begin{aligned}
J_{2} & =C \sup _{L \in \mathbb{Z}} 2^{-L \lambda p_{1}} \\
& \cdot \sum_{k=-\infty}^{L} 2^{k \alpha p_{1}}\left(\sum_{j=k-1}^{\infty}\left\|\left[b, T_{\Omega, \sigma}\right]\left(f_{j}\right) \chi_{k}\right\|_{L^{q_{2} \cdot(\cdot)}\left(\mathbb{R}^{n}\right)}\right)^{p_{1}} \\
& \leq \underset{L \in \mathbb{Z}}{C \sup } 2^{-L \lambda p_{1}} \sum_{k=-\infty}^{L} 2^{k \alpha p_{1}}\left(\sum_{j=k-1}^{\infty}\left\|f_{j}\right\|_{L^{q_{1}(\cdot)}\left(\mathbb{R}^{n}\right)}\right)^{p_{1}} \\
& =C \sup _{L \in \mathbb{Z}} 2^{-L \lambda p_{1}} \\
& \cdot \sum_{k=-\infty}^{L}\left(\sum_{j=k-1}^{\infty} 2^{(k-j) \alpha} 2^{j \alpha}\left\|f_{j}\right\|_{L^{q_{1} \cdot(\cdot)}\left(\mathbb{R}^{n}\right)}\right)^{p_{1}} .
\end{aligned}
$$

If $0<p_{1} \leq 1$, then we have

$$
\begin{aligned}
J_{2} \leq C \sup _{L \in \mathbb{Z}} 2^{-L \lambda p_{1}} \sum_{k=-\infty}^{L} \sum_{j=k-1}^{\infty} 2^{(k-j) \alpha p_{1}} 2^{j \alpha p_{1}}\left\|f_{j}\right\|_{L^{q_{1} \cdot(\cdot)}\left(\mathbb{R}^{n}\right)}^{p_{1}} \\
\leq C \sup _{L \in \mathbb{Z}} 2^{-L \lambda p_{1}} \sum_{k=-\infty}^{L} \sum_{j=k-1}^{L-1} 2^{(k-j) \alpha p_{1}} 2^{j \alpha p_{1}}\left\|f_{j}\right\|_{L^{q_{1}(\cdot)}\left(\mathbb{R}^{n}\right)}^{p_{1}} \\
\quad+C \sup _{L \in \mathbb{Z}} 2^{-L \lambda p_{1}} \sum_{k=-\infty}^{L} \sum_{j=L}^{\infty} 2^{(k-j) \alpha p_{1}} 2^{j \alpha p_{1}}\left\|f_{j}\right\|_{L^{q_{1} \cdot(\cdot)}\left(\mathbb{R}^{n}\right)}^{p_{1}} \\
=: J_{21}+J_{22} .
\end{aligned}
$$

For $J_{21}$, we have

$$
\begin{aligned}
J_{21} & \leq C \sup _{L \in \mathbb{Z}} 2^{-L \lambda p_{1}} \sum_{j=-\infty}^{L-1} 2^{j \alpha p_{1}}\left\|f_{j}\right\|_{L^{q_{1}(\cdot)}\left(\mathbb{R}^{n}\right)}^{p_{1}} \sum_{k=-\infty}^{j+1} 2^{(k-j) \alpha p_{1}} \\
& \leq C \sup _{L \in \mathbb{Z}} 2^{-L \lambda p_{1}} \sum_{j=-\infty}^{L-1} 2^{j \alpha p_{1}}\left\|f_{j}\right\|_{L^{q_{1}(\cdot)}\left(\mathbb{R}^{n}\right)}^{p_{1}} \\
& \leq C\|f\|_{M \dot{K}_{q_{1}(\cdot)}^{\alpha, p_{1}}\left(\mathbb{R}^{n}\right)}^{p^{n}}
\end{aligned}
$$

For $J_{22}$, by $0<\lambda<\alpha$ we have

$$
\begin{aligned}
& J_{22}=C \sup _{L \in \mathbb{Z}} 2^{-L \lambda p_{1}} \sum_{k=-\infty}^{L} \sum_{j=L}^{\infty} 2^{(k-j) \alpha p_{1}} 2^{j \alpha p_{1}}\left\|f_{j}\right\|_{L^{q_{1} \cdot(\cdot)}\left(\mathbb{R}^{n}\right)}^{p_{1}} \leq C \\
& \cdot \sup 2^{-L \lambda p_{1}} \\
& \cdot \sum_{k=-\infty}^{L} \sum_{j=L}^{\infty} 2^{(k-j) \alpha p_{1}} 2^{j \lambda p_{1}} 2^{-j \lambda p_{1}}\left(\sum_{m=-\infty}^{j} 2^{m \alpha p_{1}}\left\|f_{m}\right\|_{L^{q_{1}(\cdot)}\left(\mathbb{R}^{n}\right)}^{p_{1}}\right) \\
& \leq C \sup _{L \in \mathbb{Z}} 2^{-L \lambda p_{1}} \sum_{k=-\infty}^{L} \sum_{j=L}^{\infty} 2^{(k-j) \alpha p_{1}} 2^{j \lambda p_{1}}\|f\|_{\left.M K_{q_{1}(\cdot)}^{p_{1}} p^{n}\right)}^{p_{1}} \\
& =C\|f\|_{M \dot{K}_{q_{1}(\cdot)}^{\alpha, p_{1}\left(\mathbb{R}^{n}\right)}}^{p_{L}} \sup _{L \in \mathbb{Z}} 2^{-L \lambda p_{1}} \sum_{k=-\infty}^{L} 2^{k \alpha p_{1}} \sum_{j=L}^{\infty} 2^{j(\lambda-\alpha) p_{1}} \\
& \leq C\|f\|_{M \dot{K}_{q_{1}(\cdot)}^{\alpha, p_{1}}}^{p_{\mathbb{R}^{n}}} \sup _{L \in \mathbb{Z}} 2^{-L \lambda p_{1}} 2^{L \alpha p_{1}} 2^{L(\lambda-\alpha) p_{1}} \\
& =C\|f\|_{M K_{\left.q_{1}()^{\prime}\right)}^{\left.\alpha, \alpha p_{1}\right)}}^{p_{1}} .
\end{aligned}
$$

If $1<p_{1}<\infty$, noting $\lambda<\alpha$, we can take a constant $\eta>1$ so that $\lambda-\alpha / \eta<0$. By the Hölder inequality we have

$$
\begin{aligned}
J_{2} \leq & C \sup _{L \in \mathbb{Z}} 2^{-L \lambda p_{1}} \\
& \cdot \sum_{k=-\infty}^{L}\left(\sum_{j=k-1}^{\infty} 2^{(k-j) \alpha p_{1} / \eta} 2^{j \alpha p_{1}}\left\|f_{j}\right\|_{L^{q_{1}(\cdot)}\left(\mathbb{R}^{n}\right)}^{p^{n}}\right) \\
& \cdot\left(\sum_{j=k-1}^{\infty} 2^{(k-j) \alpha p_{1}^{\prime}(\eta-1) / \eta}\right)^{p_{1} / p_{1}^{\prime}} \leq C \sup _{L \in \mathbb{Z}} 2^{-L \lambda p_{1}} \\
& \cdot \sum_{k=-\infty}^{L} \sum_{j=k-1}^{\infty} 2^{(k-j) \alpha p_{1} / \eta} 2^{j \alpha p_{1}}\left\|f_{j}\right\|_{L^{q_{1} \cdot(\cdot)}\left(\mathbb{R}^{n}\right)}^{p_{1}} \\
& \leq C \sup _{L \in \mathbb{Z}} 2^{-L \lambda p_{1}} \\
& \cdot \sum_{k=-\infty}^{L} \sum_{j=k-1}^{L-1} 2^{(k-j) \alpha p_{1} / \eta} 2^{j \alpha p_{1}}\left\|f_{j}\right\|_{L^{q_{1} \cdot(\cdot)}\left(\mathbb{R}^{n}\right)}^{p_{1}} \\
& +C \sup _{L \in \mathbb{Z}} 2^{-L \lambda p_{1}} \sum_{k=-\infty}^{L} \sum_{j=L}^{\infty} 2^{(k-j) \alpha p_{1} / \eta} 2^{j \alpha p_{1}}\left\|f_{j}\right\|_{L^{q_{1}(\cdot)}\left(\mathbb{R}^{n}\right)}^{p_{1}} \\
& =: J_{23}+J_{24} \cdot
\end{aligned}
$$

For $J_{23}$, we have

$$
\begin{aligned}
& J_{23} \\
& \quad C \sup _{L \in \mathbb{Z}} 2^{-L \lambda p_{1}} \sum_{j=-\infty}^{L-1} 2^{j \alpha p_{1}}\left\|f_{j}\right\|_{L^{q_{1}(\cdot)}\left(\mathbb{R}^{n}\right)}^{p_{1}} \sum_{k=-\infty}^{j+1} 2^{(k-j) \alpha p_{1} / \eta}
\end{aligned}
$$




$$
\begin{aligned}
& \leq C \sup _{L \in \mathbb{Z}} 2^{-L \lambda p_{1}} \sum_{j=-\infty}^{L-1} 2^{j \alpha p_{1}}\left\|f_{j}\right\|_{L^{q_{1}(\cdot)}\left(\mathbb{R}^{n}\right)}^{p_{1}} \\
& \leq C\|f\|_{M \dot{K}_{q_{1}(\cdot)}^{\alpha, p_{1}}\left(\mathbb{R}^{n}\right)}^{p_{1}}
\end{aligned}
$$

For $J_{24}$, by $0<\lambda<\alpha / \eta$ we have

$$
\begin{aligned}
& J_{24}=C \sup _{L \in \mathbb{Z}} 2^{-L \lambda p_{1}} \sum_{k=-\infty}^{L} \sum_{j=L}^{\infty} 2^{(k-j) \alpha p_{1} / \eta} 2^{j \alpha p_{1}}\left\|f_{j}\right\|_{L^{q_{1}(\cdot)}\left(\mathbb{R}^{n}\right)}^{p_{1}} \\
& \leq C \sup _{L \in \mathbb{Z}} 2^{-L \lambda p_{1}} \sum_{k=-\infty}^{L} \sum_{j=L}^{\infty} 2^{(k-j) \alpha p_{1} / \eta} 2^{j \lambda p_{1}}\|f\|_{M \dot{K}_{q_{1}(\cdot)}^{\alpha, p_{1}}\left(\mathbb{R}^{n}\right)}^{p_{1}} \\
& \left.=C\|f\|_{M \dot{K}_{q_{1}(\cdot)}^{\alpha, p_{1}}}^{p_{1}} \mathbb{R}^{n}\right) \sup _{L \in \mathbb{Z}} 2^{-L \lambda p_{1}} \sum_{k=-\infty}^{L} 2^{k \alpha p_{1} / \eta} \sum_{j=L}^{\infty} 2^{j(\lambda-\alpha / \eta) p_{1}} \\
& \left.\leq C\|f\|_{M \dot{K}_{q_{1}(\cdot)}^{\alpha, p_{1}}}^{p_{1}} \mathbb{R}^{n}\right) \sup _{L \in \mathbb{Z}} 2^{-L \lambda p_{1}} 2^{L \alpha p_{1} / \eta} 2^{L(\lambda-\alpha / \eta) p_{1}} \\
& =C\|f\|_{M \dot{K}_{q_{1}(\cdot)}^{\alpha, p_{1}}}^{p_{1}}\left(\mathbb{R}^{n}\right)
\end{aligned}
$$

Thus, by (52), (61), (62), and (64)-(69) we complete the proof of Theorem 18 .

Remark 20. In [28], the authors pointed out BMO C $\bigcap_{q>1} \mathrm{CMO}^{q}$, so we will consider the corresponding results on $\mathrm{CMO}$ space in the following paper.

\section{Conflicts of Interest}

The authors declare that there are no conflicts of interest regarding the publication of this paper.

\section{Acknowledgments}

This work was partially supported by NSF of China (Grant no. 11671185), Shandong Provincial Natural Science Foundation, China (Grant no. ZR2017MA041), and China Postdoctoral Science Foundation funded project (Grant no. 2016M601105).

\section{References}

[1] C. B. Morrey, "On the solutions of quasi-linear elliptic partial differential equations," Transactions of the American Mathematical Society, vol. 43, no. 1, pp. 126-166, 1938.

[2] J. Alvarez, M. Guzmán-Partida, and J. Lakey, "Spaces of bounded $\lambda$-central mean oscillation, morrey spaces, and $\lambda$ central carleson measures," Collectanea Mathematica, vol. 51, no. 1, pp. 1-47, 2000.

[3] C. Herz, "Lipschitz spaces and Bernstein's theorem on absolutely convergent Fourier transforms," Journal of Mathematics and Mechanics, vol. 18, pp. 283-324, 1968.

[4] S. Lu, D. Yang, and G. Hu, Herz Type Spaces and Their Applications, Science Press, Beijing, China, 2008.
[5] S. Lu and L. Xu, "Boundedness of rough singular integral operators on the homogeneous Morrey-Herz spaces," Hokkaido Mathematical Journal, vol. 34, no. 2, pp. 299-314, 2005.

[6] O. Kováčik and J. Rákosník, "On spaces $L^{p(x)}$ and $W^{k, p(x), "}$ Czechoslovak Mathematical Journal, vol. 41, no. 116, pp. 592-618, 1991.

[7] D. V. Cruz-Uribe and A. Fiorenza, Variable Lebesgue Spaces: Foundations and Harmonic Analysis, Applied and Numerical Harmonic Analysis, Springer, Heidelberg, Germany, 2013.

[8] L. Diening, P. Harjulehto, P. Hästö, and M. Ružicka, Lebesgue and Sobolev Spaces with variable Exponents, vol. 2017 of Lecture Notes in Math, Springer, Heidelberg, Germany, 2011.

[9] M. Ružička, Electrorheological Fluids: Modeling and Mathematical Theory, Springer, Berlin, Germany, 2000.

[10] Y. Chen, S. Levine, and M. Rao, "Variable exponent, linear growth functionals in image restoration," SIAM Journal on Applied Mathematics, vol. 66, no. 4, pp. 1383-1406, 2006.

[11] P. Harjulehto, P. Hästö, U. V. Lê, and M. Nuortio, "Overview of differential equations with non-standard growth," Nonlinear Analysis. Theory, Methods \& Applications. An International Multidisciplinary Journal, vol. 72, no. 12, pp. 4551-4574, 2010.

[12] M. Izuki, "Herz and amalgam spaces with variable exponent, the Haar wavelets and greediness of the wavelet system," East Journal on Approximations, vol. 15, no. 1, pp. 87-109, 2009.

[13] M. Izuki, "Boundedness of vector-valued sublinear operators on Herz-Morrey spaces with variable exponent," Mathematical Sciences Research Journal, vol. 13, no. 10, pp. 243-253, 2009.

[14] Y. Mizuta, T. Ohno, and T. Shimomura, "Boundedness of maximal operators and Sobolev's theorem for non-homogeneous central Morrey spaces of variable exponent," Hokkaido Mathematical Journal, vol. 44, no. 2, pp. 185-201, 2015.

[15] B. Muckenhoupt and R. L. Wheeden, "Weighted norm inequalities for singular and fractional integrals," Transactions of the American Mathematical Society, vol. 161, pp. 249-258, 1971.

[16] J. Tan and Z. Liu, "Some boundedness of homogeneous fractional integrals on variable exponent function spaces," Acta Mathematica Sinica. Chinese Series, vol. 58, no. 2, pp. 309-320, 2015.

[17] S. Kong, M. Saif, and B. Liu, "Observer design for a class of nonlinear fractional-order systems with unknown input," Journal of the Franklin Institute. Engineering and Applied Mathematics, vol. 354, no. 13, pp. 5503-5518, 2017.

[18] C. Capone, D. Cruz-Uribe, and A. Fiorenza, "The fractional maximal operator and fractional integrals on variable $L^{p}$ spaces," Revista Matemática Iberoamericana, vol. 23, no. 3, pp. 743-770, 2007.

[19] M. Izuki, "Boundedness of sublinear operators on Herz spaces with variable exponent and application to wavelet characterization," Analysis Mathematica, vol. 36, no. 1, pp. 33-50, 2010.

[20] E. Nakai and Y. Sawano, "Hardy spaces with variable exponents and generalized Campanato spaces," Journal of Functional Analysis, vol. 262, no. 9, pp. 3665-3748, 2012.

[21] H. Wang and Z. Liu, "Some characterizations of Herz-type Hardy spaces with variable exponent," Annals of Functional Analysis, vol. 6, no. 2, pp. 224-233, 2015.

[22] A. Abdalmonem, O. Abdalrhman, and S. Tao, "The boundedness of fractional integral with variable kernel on variable exponent herz-morrey spaces," Journal of Applied Mathematics and Physics, vol. 04, no. 04, pp. 787-795, 2016.

[23] C. Segovia and J. L. Torrea, "Higher order commutators for vector-valued Calderón-Zygmund operators," Transactions of 
the American Mathematical Society, vol. 336, no. 2, pp. 537-556, 1993.

[24] Y. Ding, "Weighted boundedness for commutators of integral operators of fractional order with rough kernels," Beijing Shifan Daxue Xuebao, vol. 32, no. 2, pp. 157-161, 1996 (Chinese).

[25] Y. Ding and S. Lu, "Higher order commutators for a class of rough operators," Arkiv för Matematik, vol. 37, no. 1, pp. 33-44, 1999.

[26] D. Cruz-Uribe, A. Fiorenza, J. M. Martell, and C. Pérez, "The boundedness of classical operators on variable $L^{p}$ spaces," Annales Academice Scientiarum Fennica Mathematica, vol. 31, no. 1, pp. 239-264, 2006.

[27] M. Izuki, "Boundedness of commutators on Herz spaces with variable exponent," Rendiconti del Circolo Matematico di Palermo. Second Series, vol. 59, no. 2, pp. 199-213, 2010.

[28] Z. W. Fu, Y. Lin, and S. Z. Lu, " $\lambda$-central BMO estimates for commutators of singular integral operators with rough kernels," Acta Mathematica Sinica (English Series), vol. 24, no. 3, pp. 373386, 2008. 


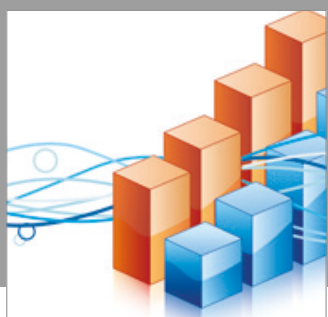

Advances in

Operations Research

vatersals

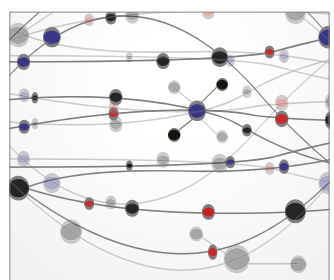

\section{The Scientific} World Journal
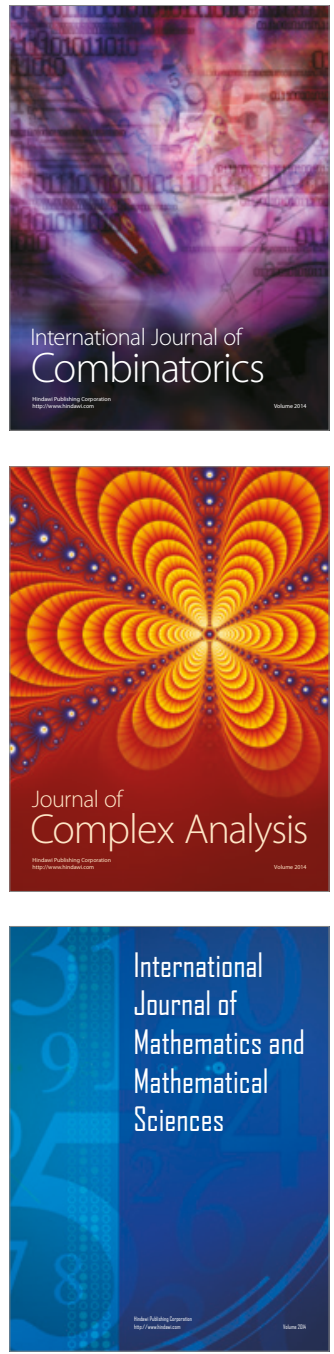
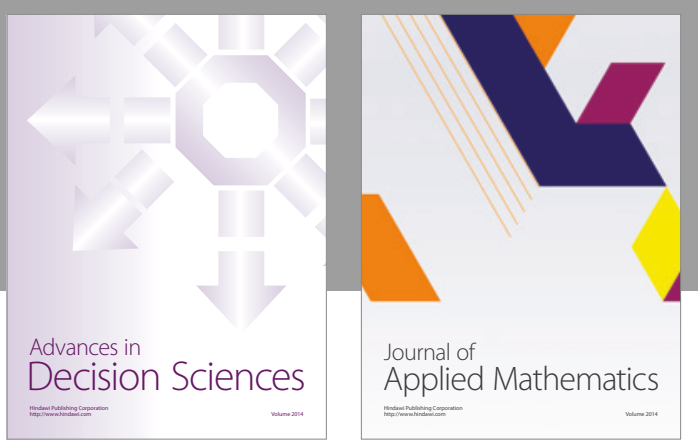

Algebra

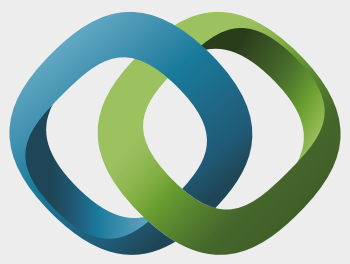

\section{Hindawi}

Submit your manuscripts at

https://www.hindawi.com
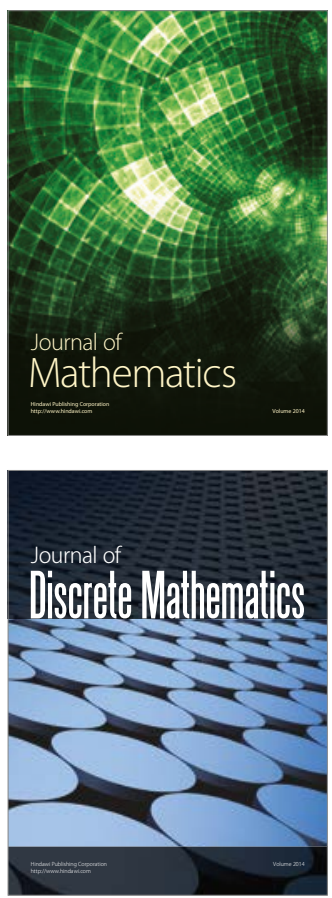

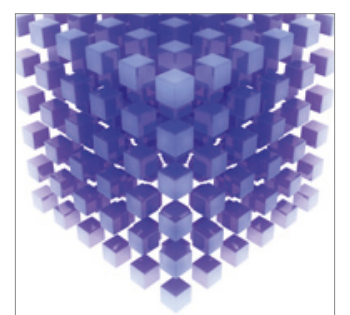

Mathematical Problems in Engineering
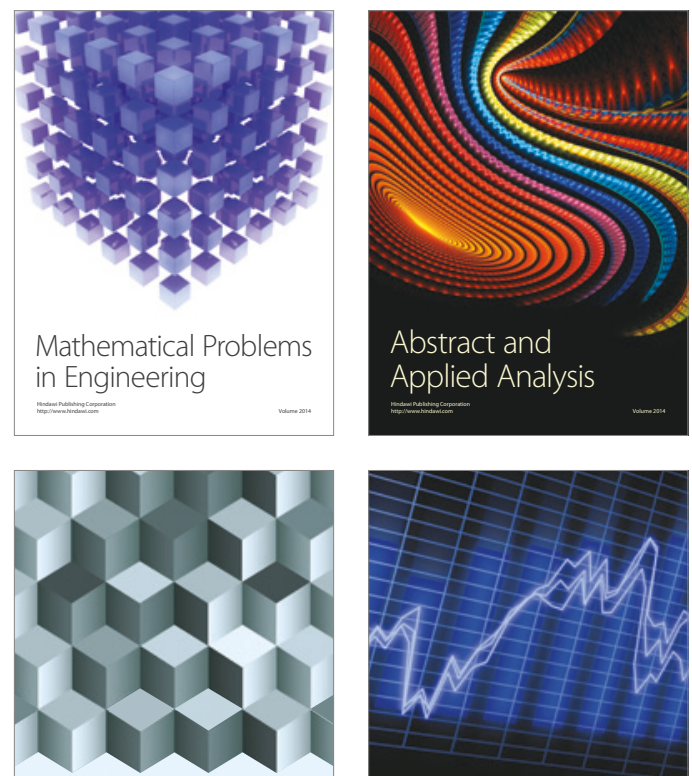

Journal of

Function Spaces

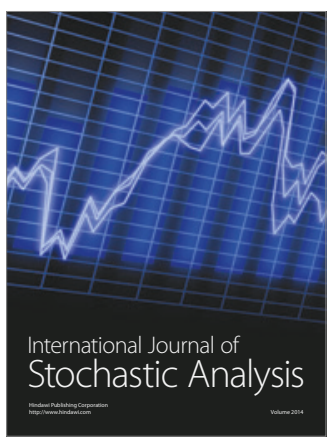

Probability and Statistics
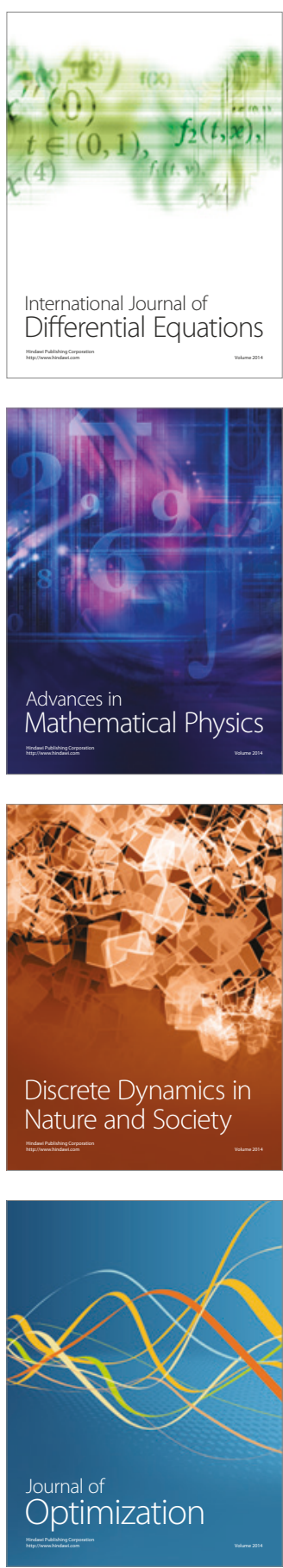